\title{
Exocyst Inactivation in Urothelial Cells Disrupts Autophagy and Activates non-canonical
}

\section{NF-KB}

Michael A. Ortega ${ }^{1,2}$, Ross K. Villiger², Malia Harrison-Chau², Suzanna Lieu², Kadee-Kalia Tamashiro ${ }^{2}$, Amanda J. Lee ${ }^{2,3}$, Brent A. Fujimoto², Geetika Y. Patwardhan², Joshua Kepler² \& Ben Fogelgren*2

${ }^{1}$ Center for Biomedical Research at The Queen's Medical Center, Honolulu, Hawaii 96813;

${ }^{2}$ Department of Anatomy, Biochemistry and Physiology, John A. Burns School of Medicine, University of Hawaii at Manoa, Honolulu, Hawaii 96813; ${ }^{3}$ Math and Sciences Department, Kapiolani Community College, Honolulu, Hawaii 96816.

${ }^{*}$ Corresponding Author:

Ben Fogelgren

Email address: fogelgre@hawaii.edu

Keywords: autophagy / exocyst / Fn14 / NF-kB / urothelium 


\begin{abstract}
Ureter obstruction is a highly prevalent event during embryonic development and is a major cause of pediatric kidney disease. We have reported that ureteric bud-specific ablation of the exocyst Exoc5 subunit in late-murine gestation results in failure of urothelial stratification, cell death, and complete ureter obstruction. However, the mechanistic connection between disrupted exocyst activity, urothelial cell death, and subsequent ureter obstruction was unclear. Here, we report that inhibited urothelial stratification does not drive cell death during ureter development. Instead, we demonstrate that the exocyst plays a critical role in autophagy in urothelial cells, and that disruption of autophagy activates a urothelial NF-kB stress response. Impaired autophagy first provokes canonical NF-KB activity which is progressively followed by increasing non-canonical NF-KB activity and cell death if the stress remains unresolved. Furthermore, we demonstrate that ureter obstructions can be completely rescued in Exoc5 conditional knockout mice by administering a single dose of pan-caspase inhibitor z-VAD-FMK at E16.5 prior to urothelial cell death. Taken together, ablation of Exoc5 disrupts autophagic stress response and activates progressive NF-KB signaling which promotes obstructive uropathy.
\end{abstract}




\section{Introduction}

Ureter obstruction during fetal development is a common cause of children being born with congenital anomalies of the kidney and urinary tract (CAKUT) (Chua et al, 2019; Johansen et al, 2021; Verbitsky et al, 2019). The most prevalent site of obstruction occurs at the ureteropelvic junction (UPJ), where the renal pelvis transitions into the upper ureter, resulting in restricted urine flow that can cause lasting kidney damage (Roth et al, 2002). Whether the obstruction resolves naturally or is surgically corrected, as many as $70 \%$ of patients with congenital obstructive uropathy $(\mathrm{COU})$ will develop a gradual loss of kidney function and progress to end-stage renal disease by the age of 20 (Chevalier et al, 2010; Craven et al, 2007; Mesrobian \& Mirza, 2012; Miklovicova et al, 2008). However, there remains a limited understanding of the mechanisms that govern the embryonic onset of COU.

Several mouse models implicate urothelium abnormalities as a major driver behind ureter obstructions (Jackson et al, 2020). The urothelium is a specialized stratified epithelium that functions as a urine permeability barrier along the upper urinary tract and bladder. We have previously reported that conditional knock out of the exocyst Exoc5 gene in ureteric bud cells disrupts the urothelial stratification process in embryonic ureters, which subsequently triggers cell death between E16.5-E17.5 (Fogelgren et al, 2015). Cell death is followed by a wound healing response that causes UPJ lumen obliteration through the activation and expanse of myofibroblasts (Fogelgren et al., 2015; Lee et al, 2016). The Exoc5 conditional knockout was achieved using the Ksp-cadherin Cre mouse strain $\left(\mathrm{Cre}^{\mathrm{Ksp}}\right)$, which we demonstrated to be active

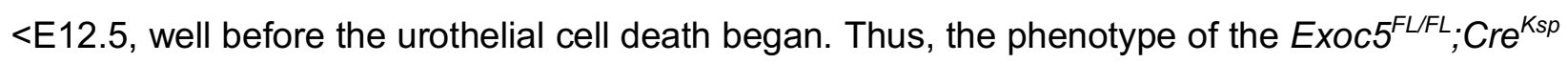
mouse (Exoc5-CKO) is similar to intrinsic human congenital UPJ obstructions and is a valuable model for investigating the underlying mechanisms of ureter obstruction.

EXOC5 is a core component of the highly-conserved octameric exocyst protein complex (made up of proteins EXOC1-8) that mediates the targeting and docking of intracellular vesicles (Ahmed et al, 2018; Heider et al, 2016; Mei et al, 2018; Morin et al, 2010; TerBush et al, 1996). Since it 
associates with specific vesicles by members of the Rab GTPases family, the exocyst is often classified as a Rab effector complex. The localization and assembly of the exocyst holocomplex is guided in part by members of the Ras superfamily of small GTPases such as RALA and RALB, which regulate distinct biological processes by interacting with different exocyst subunits. As an example, the RALA-EXOC2 complex mediates trafficking of proteins to the basolateral membrane in epithelial cells (Moskalenko et al, 2002), while the RALB-EXOC2 complex can directly activate innate immunity and restrict apoptosis by engaging the IkB kinase family member TBK1 (Chien et al, 2006; Shipitsin \& Feig, 2004). Alternatively, RALB-EXOC8 can promote autophagy by acting as an assembly scaffold for ULK1 and Beclin1-VPS34 during nutrient deprivation (Bodemann et al, 2011; Martin et al, 2014; Singh et al, 2019). The ubiquitylation state of RALB at Lys47 was shown to drive RALB towards mediating the exocyst complex into activating innate immunity or autophagy, thereby implicating the exocyst as a critical moderator of cellular stress response (Simicek et al, 2013).

Studies in Drosophila have revealed that exocyst-mediated autophagy is tissue-specific and context dependent (Mohseni et al, 2009; Tracy et al, 2016). Autophagy is an evolutionarily conserved lysosomal degradation process known to be highly active during differentiation and development (Mizushima \& Levine, 2010). This pathway is critical for many physiological events, such as responding to cellular stress by maintaining homeostasis through the clearance of damaged organelles and proteins (Kroemer et al, 2010; Tang et al, 2020). Interestingly, autophagy has been reported to either inhibit or promote cell death in a cell- and injury- specific manner, but autophagy appears to be dispensable for mammalian ureter and kidney development (Goodall et al, 2016; Gump et al, 2014; Komatsu et al, 2005; Kuma et al, 2004; Nezis et al, 2010; Thorburn et al, 2014). However, several mouse models utilizing tissue-specific autophagy related gene (ATG) knockout demonstrate that deficiencies in autophagy promote progressive pathology by limiting the organism's ability to respond to stress (Bechtel et al, 2013; Hartleben et al, 2010; Kim et al, 2012; Takahashi et al, 2012). 
Here, we report that ureter obstruction in Exoc5 CKO mice is not the result failed urothelial stratification but instead occurs because of the unresolved urothelial cell death. Furthermore, the fibroproliferative wound healing reaction that obliterates the ureter lumen is not activated ex vivo in the absence of urine. Autophagy is impaired after urothelial Exoc5 ablation, which triggers an increasing prevalence of the TNF superfamily receptor Fn14, a potent activator of the noncanonical NF-KB pathway. Our data demonstrate that inhibiting autophagy in urothelial cells provokes an initial p65 canonical NF-KB response that is progressively followed by a secondary p52 non-canonical NF-kB response and then cell death. Lastly, we demonstrate that the Exoc5 CKO ureter development is completely rescued with a single administration of pan-caspase zVAD-fmk inhibitor at E16.5. Taken together, these data demonstrate impaired exocyst-mediated autophagy in urothelial cells activates progressive NF-KB signaling that enhanced cell death and promotes the onset of COU.

\section{Results}

Differentiating urothelial cells initiate cell death independent of failed stratification. Abnormal urothelial differentiation has been implicated in mouse models and patients with

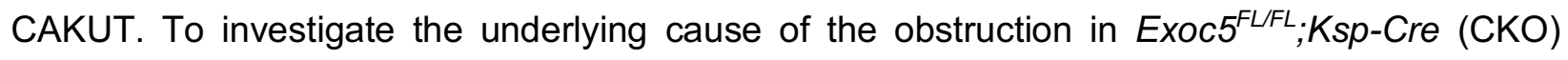
embryonic ureters, we developed an ex vivo ureter explant culture system. Wild type C57BL/6J mouse embryonic ureters were collected at E15.5 and cultured for 72 hours on semi-permeable supports at the air-liquid interface (Figure 1A). In this system, the explant urothelium starts as a single epithelial monolayer, as shown by immunostaining for E-cadherin (green) and smooth muscle actin (SMA, red) $(E 15.5, t=0)$, and successfully differentiates into a stratified epithelium with strong uroplakin staining on the luminal surface $(\mathrm{E} 18.5, \mathrm{t}=72 \mathrm{hrs})$. Uroplakins are only produced by superficial cells, thus indicating successful urothelial differentiation in an ex vivo setting over a time course similar to in vivo ureter development. After 72 hours, the explant ureters showed a significant increase in overall growth and continued to have regular peristalsis, 
indicating the successful maturation of the smooth muscle layer that surrounds the ureters (Figure 1B).

Upon establishing this ex vivo ureter model, we wanted to determine if failed stratification was the underlying cause of urothelial cell death observed in Exoc5 CKO mice. To test this, we treated E15.5 explant ureters with either vehicle control or retinoic acid receptor inverse agonist BMS 493 for 72 hours, since retinoic acid signaling is known to be necessary for urothelial differentiation (Gandhi et al, 2013). Explant ureters treated with BMS 493 were unable to differentiate a multilayered epithelium, as shown with H\&E staining and E-cadherin/SMA immunohistochemistry (Figure 1C). The BMS 493-treated ureters also completely lacked uroplakin expression (Figure 1C, Figure EV1), however, there were no signs of urothelial cell death or lumen obliteration. Realtime qPCR analysis of BMS 493-treated samples showed Ppar $\gamma$, a downstream target of RA, was also strongly down regulated, confirming that BMS 493 successfully disrupted the RA signaling pathway and urothelial differentiation (Figure EV1).

Next, we wanted to use this ureter explant model to determine if the Exoc5 CKO urothelial cells still underwent cell death, and if the fibroproliferative obstruction would be induced, in the absence of urine flow. For this, we cultured Exoc5 CKO and littermate control E15.5 explants for 72 hours, followed by immunohistochemistry. As with the wild type ureter explants, control ureter explants showed the presence of a normal multilayered urothelium after 72 hours, while CKO ureters displayed a disrupted urothelium with urothelial cells sloughing off and entering the luminal space as seen with E-cadherin (green) (Figure 1D). As described in vivo (Fogelgren et al., 2015; Lee et al., 2016), Exoc5 CKO ureter explants also showed no uroplakin expression, indicating the urothelial progenitors failed to differentiate into superficial cells. However, unlike the pathology of Exoc5 CKO ureters in vivo, the distribution of collagen IV indicated an intact basement membrane, with no expansion of mesenchymal cells, suggesting a fibroproliferative response was not activated as a result of the urothelial cell death. Taken together, these results suggest that the 
epithelial cells comprising the urothelial progenitor monolayer initiate cell death independently of failed differentiation or the presence of urine.

NF-KB activator Fn14 is highly upregulated in Exoc5 CKO ureters. To identify molecules potentially involved in the urothelial cell death event in Exoc5 CKO ureters, we performed gene profiling on microdissected E16.5 ureter RNA samples using Affymetrix Clariom D GeneChip microarrays. As expected based on our previously reported data (Fogelgren et al., 2015), we measured strongly decreased expression of uroplakin genes in Exoc5 CKO ureters (Figure 2A). One of the most upregulated individual genes was Fn14 (Tnfrsf12a) which is a TNF superfamily receptor known to play a role in both canonical and non-canonical NF-kB signaling (Figure 2A). Fn14 has only one known ligand, a cytokine named TWEAK (tumor necrosis factor-like weak inducer of apoptosis), and has been shown to be a stress-response gene in many tissues (MuñozGarcía et al, 2006; Nagy et al, 2021; Peng et al, 2018; Unudurthi et al, 2020; Zhao et al, 2007). Fn14 is often strongly upregulated after cell damage or oncogenic transformation (Johnston et al, 2015), however there are no published reports of its activity in urothelial cells. We then performed KEGG pathway analysis which implicated NF-KB signaling among the most upregulated pathways in response to Exoc5 ablation (Figure 2B). qPCR validation confirmed the loss of uroplakin expression in these Exoc5 CKO samples and that expression of Fn14 and its ligand TWEAK were both increased by more than 30 fold at E16.5 (Figure 2C). Since Fn14 has a relatively short halflife of $\sim 74 \mathrm{~min}$ (Gurunathan et al, 2014), we performed western blotting on isolated E16.5 and at E17.5 ureters to measure protein levels. Fn14 maintained relatively low protein levels at E16.5, however, there was a strong buildup of Fn14 in E17.5 Exoc5 CKO ureters, indicating that Fn14 was elevated during this period of stress (Figure 2D). The degree of Fn14 increase in E17.5 Exoc5 CKO ureters varied but was consistently and significantly higher compared to other genotypes (Figure 2E). In order to determine where Fn14 was being expressed, we performed immunohistochemistry and identified Fn14 was strongly upregulated in the urothelium, but also in 
the underlying mesenchymal cells (Figure $2 \mathrm{~F}$ ). These data demonstrate that the NF-KB signaling activator Fn14 robustly responds to stress induced by Exoc5 CKO during ureter development.

LC3 accumulates in Exoc5 CKO urothelium. KEGG analysis of the most downregulated pathways revealed several metabolic mechanisms as being significantly perturbed following Exoc5 ablation (Figure 2G). Given that the exocyst has been implicated in the initiation process of autophagic stress response (Bodemann et al., 2011), we reasoned that exocyst-mediated autophagy may be deficient in the urothelium and contributing to the downregulation of these metabolic pathways. Since LC3 is known to accumulate when autophagy is impaired (Runwal et al, 2019), we tested this by performing immunohistochemistry of the LC3 protein in E17.5 Exoc5 CKO ureters. While control ureters showed no obvious abnormalities and only low levels of LC3, Exoc5 CKO ureters displayed a strong accumulation of LC3 in the urothelium while the surrounding smooth muscle layer did not show significant increase (Figure $2 \mathrm{H}$ ). It was noted that the Exoc5 CKO urothelium showed LC3 puncta accumulation (Figure I, arrow), which is commonly observed when LC3 is not degraded from active autophagy.

Inhibiting exocyst function causes impaired autophagy in urothelial cells. Though interactions between exocyst and autophagy related genes (ATG) have been previously reported, the functional consequence of perturbing this relationship in urothelial cells remains unclear. Here, we used $100 \mu \mathrm{M}$ endosidin-2 (ES2) to inhibit the binding of exocyst subunits to the EXOC7 exocyst subunit (Zhang et al, 2016). ES2 treatment of primary human urothelial (pHUC) cells caused progressive vesicle accumulation over 24 hours, which was visible even in phase contrast (Figure $3 A, B)$. To determine if ES2 vesicle accumulation was the result of impaired autophagy in pHUCs, we performed immunocytochemistry following 24h ES2 treatment and observed that EXOC5 and ATG5 colocalized in control pHUCs, but ATG5 puncta were highly decreased after ES2 treatment (Figure 3C). Next, we performed western blotting of $24 \mathrm{~h}$ ES2-treated pHUCs to measure protein levels of classic autophagy markers. Here, we measured a decrease in ATG5 levels, and significant increase in LC3II/I ratio and p62 accumulation after ES2 treatment (Figure 3 D,E). To 
test if the exocyst and ATGs directly associated in human urothelial cells, we performed immunoprecipitation of EXOC4 followed by western blotting for ATG7 using SV-HUC-1 immortalized urothelial cells and observed positive pulldown (Figure 3F). These data show that exocyst interacts with ATGs and that inhibiting trafficking disrupts the process of autophagy.

To further demonstrate that inhibiting exocyst function had a detrimental effect on autophagy in urothelial cells, we ablated Exoc5 in the adult bladder urothelium by crossing Exoc5 ${ }^{F L / F L}$ with Upk3a-GCE mice, which express both tamoxifen-activated Cre and GFP under the uroplakin-3a promoter (Honeycutt et al, 2015). In parallel, tdTomato Cre-reporter mice were crossed with Upk3a-GCE mice to assess Cre recombinase activity and specificity after tamoxifen treatments (Figure 4A). The Exoc5 $5^{F L F L} ;$ Upk3a-GCE mice were treated with tamoxifen at 6-8 weeks of age to generate induced urothelial Exoc5 knockout (Exoc5-iUKO) mice, with control mice defined as Exoc5 $5^{F L / F L}$ mice treated with an identical regimen of tamoxifen. The Exoc5-iUKO survived with no gross abnormalities until euthanized 4 weeks later for bladder histology and electron microscopy. Scanning electron microscopy on the Exoc5-iUKO luminal bladder surface did not reveal any noticeable abnormalities in uroplakin plaque structures, and H\&E histology was unremarkable. However, transmission electron microscopy (TEM) on Exoc5-iUKO bladder sections revealed a significant buildup of lysosomes as marked by electron dense organelles, which was similar to observations found in age-related lysosomal disease models (Figure 4B) (Truschel et al, 2018). To test for impairments in autophagy in these Exoc5-iUKO urothelial cells, we performed immunofluorescent detection of LC3 on bladder sections and observed classical large LC3 puncta formation at a highly increased instance over those seen in controls (Figure 4C,D). Taken together, these data indicate that the exocyst biochemically interacts with ATGs in urothelial cells and that inhibiting exocyst function detrimentally affects autophagy in a tissue-specific manner.

Fn14 is upregulated by inhibiting autophagy in urothelial cells. We wanted to determine if there was a causal relationship between impaired autophagy and the noncanonical NF-kB signaling observed in our microarray data. To test this, we investigated whether Fn14 responded 
to disruptions of autophagy and other forms of stress in SV-HUC-1 cells. First, we treated SVHUC-1 cells with $20 \mu \mathrm{M}$ cisplatin for 4 hours to assess the effect of cisplatin-induced DNA damage on Fn14 levels and found no significant induction of Fn14 (Figure 5A,B). Next, we exposed SVHUC-1 cells to UV $40 \mathrm{~J} / \mathrm{m}^{2}$ and found no significant Fn14 increase after 6 hours of recovery (Figure 5C,D). These results indicated that under two different forms of DNA damage induced cell stress, Fn14 was not upregulated in the measured timeframes. Next, to determine if exocyst depletion impacted SV-HUC-1 cells ability to respond to stress we generated shExoc5 stable knockdowns. We observed by western blotting that treating SV-HUC-1 cells with 200nM of autophagy inhibitor BafA1 for 24h caused a significant increase in Fn14 and that this response appeared synergistic in shExoc5 knockdown cells (Figure 5 E,F,G). 200nM BafA1 treatment of wild type SV-HUC-1 cells over a 5 day period greatly reduced colony formation (Figure $5 \mathrm{H}, \mathrm{I}$ ) with significantly less cell viablity observed after 3 days (Figure 5J). Interestingly, inhibiting autophagy with either BafA1 or VPS34 inhibitor (VPS34i) both induced vesicle accumulation that was similar to ES2-treated cells (Figure EV2). These results indicated that inhibiting autophagy with BafA1 was sufficient to induce Fn14 and promote cell death. These data suggest that EXOC5 knockdown added an additional stress in complement to the direct autophagy inhibition.

Inhibiting autophagy activates two waves of NF-KB signaling. Next, we wanted to determine if NF-KB signaling was responding to cell stress triggered by impaired autophagy in these urothelial cells. First, we performed immunocytochemistry on SV-HUC-1 cells treated with either 200nM BafA1 or $50 \mu \mathrm{M}$ VPS34i to determine if canonical or non-canonical NF-kB were being stimulated with autophagy inhibition. We measured the percentage of cells showing nuclear translocation of RelA (p65) as early as 6 hours after either BafA1 or VPS34i treatment (Figure 6A, Figure EV2), indicating that canonical NF-kB signaling was active. Nuclear translocation of RelA remained present through 48 hours, although varying levels of saturation were observed. To determine if non-canonical NF-KB was also being stimulated by autophagy inhibition, we performed a similar timecourse and measured the percentage of cells with p52 nuclear 
translocation. While p52 nuclear translocation was not significantly detected 6 hours, we found an increasing prevalence as time passed from 24 hours to 48 hours nearing the time of significant BafA1-induced cell death (Figure 6B). These data indicate that inhibiting autophagy results in canonical NF-kB activity and that prolonged treatment promotes non-canonical NF-kB activity.

While TNF superfamily receptors can activate canonical NF-kB, Fn14 is one of the few known receptors to primarily activate non-canonical NF-kB signaling. To confirm that Fn14 signaling was active in SV-HUC-1 cells, we applied its only known ligand, the cytokine TWEAK, and observed a strong increase of both p52 and RelA nuclear translocation by 6 hours (Figure 6C, Figure EV3). However, only p52 remained at significant levels by 24 and 48 hours (Figure 6D). This indicated non-canonical NF-kB signaling can be activated in human urothelial cells through the TWEAKFn14 signaling axis and that while both NF-KB pathways can be activated by TWEAK in urothelial cells, the promotion of non-canonical NF-kB signaling was sustained for longer periods. Taken together, these data indicate TWEAK-Fn14 activate canonical NF-KB in response to autophagy inhibition as initial response to stress but that a second wave of noncanonical NF-kB follows if the insult persists (Figure 6D).

z-VAD-FMK rescues cell death and ureter obstruction in Exoc5 CKO ureters. Since TWEAKFn14 and non-canonical NF-kB signaling can initiate caspase activity and cell death, we explored whether Exoc5 CKO urothelial death could be prevented in vivo with a pan-caspase inhibitor (Ikner \& Ashkenazi, 2011; Martin-Sanchez et al, 2018; Vince et al, 2008). To perform this, we administered $5 \mu \mathrm{g} / \mathrm{g}$ z-VAD-FMK by intraperitoneal injection to timed mated female mice at E16.5 and collected embryos at E18.5. Under normal conditions, the Exoc5 CKO urothelium fails to stratify and undergoes cell death between E16.5 and E17.5 with full ureter obstruction by E18.5 as the underlying mesenchyme obliterates the ureter lumen (Fogelgren et al., 2015; Lee et al., 2016) (Figure 7A). However, when administering z-VAD-FMK at E16.5, we observed no urothelial cell death and complete rescue of proper ureter formation ( $n=9 / 9$ Exoc5 CKO embryos) (Figure 7A). Furthermore, the Exoc5 CKO urothelium successfully stratified as seen by UPK3 
immunofluorescent signal in the umbrella cells (Figure 7A). These in vivo results agreed with our ex vivo explant data and previously published data (Lee et al., 2016), which indicated that obstruction was the subsequent result of widespread urothelial cell death. With the obstruction prevented, we observed that hydronephrosis was avoided in these Exoc5 CKO embryos as well (Figure 7B). In addition, while Exoc5 CKO mice typically die 8-14 hours after birth E16.5 rescue trials revealed that all z-VAD-FMK-treated Exoc5 CKO mice successfully survived to adulthood.

\section{Discussion}

While defects in membrane trafficking are well known to contribute to a breadth of human diseases it remains unclear whether or not aberrant exocyst function contributes to development defects of the urinary tract, such as COU (Coulter et al, 2020; Uhm et al, 2017; Van Bergen et al, 2020). The phenotypic mirroring of human COU by the Exoc5 CKO mouse may offer a congenital model for deconstructing the molecular mechanisms underlying the onset of obstructive uropathy. Nephrogenesis is dependent on ureteric bud branching into the metanephric mesenchyme, which starts around $\mathrm{E} 11.5$, and we have shown that Cre recombinase driven by the $K s p$-cadherin promoter is active by this time (Lee et al., 2016). Thus, Exoc5 is not required for branching morphogenesis by the ureteric bud, and the urinary tract appears morphologically normal until E17.5. However, we observed that Exoc5 ablation in the urothelium resulted in impaired autophagy and non-canonical NF-kB activity prior to cell death at E17.5. These data support a model where exocyst depletion directly impairs autophagy, and that autophagy deficiencies in turn activate progressive NF-kB signaling and eventually cell death.

The relationship between defective autophagy and NF-KB signaling is intricately tied to cellular stress and damage response. For example, deficient autophagy has been shown to activate canonical NF-KB in part through an accumulation of p62, which then serves as a scaffold for the TRAF6 and RIP1 complex that drives canonical NF-kB activation (Meng \& Cai, 2011; Wooten et al, 2005). Autophagy typically suppresses p62 accumulation, thereby guarding against 
unnecessary canonical NF-kB signaling (Mathew et al, 2009). In the data presented here, we observed LC3 and p62 accumulation when inhibiting exocyst function, which suggests a path for activating canonical NF-kB as part of a survival response in urothelial cells. More specifically, it's reasonable to believe inhibiting EXOC5 would impact the formation of the RALB-EXOC84 effector complex which would have a direct impact on the ability to initiate autophagy. The relationship between autophagy deficiency and canonical NF-KB activation was further supported by nuclear translocation of RelA when we applied BafA1. The initial canonical response makes sense for promoting cell survival in the face of cell stress. However, these data further indicated that sustained stress in these cells promotes a slower second non-canonical NF-kB response.

One way to detect defective autophagy is the accumulation of LC3, as was observed in Exoc5 CKO ureter urothelium at E17.5. This LC3 accumulation was enhanced in targeted Exoc5 ablation in adult bladder urothelium using Upk3a-GCE mice. The urothelial Exoc5 ablation presented here in the ureter and bladder highlight a tissue and time specific relationship between the exocyst and autophagy. It is likely that Exoc5 ablation in the bladder urothelium presented with larger LC3 punctae because there was a longer time frame (four weeks) for this buildup to occur. This observation would also support the hypothesis that an exocyst-mediated event initiated the stress response in the developing ureter and that the impaired autophagy contributed to NF-kB activation.

Interestingly, ablation of Exoc5 would also impact formation of the RALB-EXOC2 effector complex which directly activates TBK1. TBK1 plays a critical role in inhibiting cell death and maintaining non-canonical NF-kB by phosphorylating NF-KB-inducing kinase (NIK), which leads to NIK degradation (Chien et al., 2006; Jin et al, 2012). NIK degradation is necessary for preventing p100 processing to p52 (Xiao et al, 2001). By extension, the urothelial stress induced by Exoc5 ablation could reasonably be expected to impact RALB-EXOC2 effector complex interaction with TBK1 and thereby promote non-canonical NF-KB signaling through NIK accumulation. Since, the 
exocyst is acts as a hub moderator of these pathways, it would be necessary for future confirm these interactions in ureter development.

Our observation that non-canonical p52 acts as a slower response complements results reported by Saitoh et al. 2003, in which TWEAK was used to stimulate non-canonical NF-KB independent of TNFa in MEFs and found p52 nuclear translocation was delayed in response when compared to canonical RelA nuclear translocation (Saitoh et al, 2003). Under these conditions, canonical RelA responded first but was followed by non-canonical p52 after 8 hours, whereafter p52 remained in the nucleus for at least 24 hours (Saitoh et al., 2003). Depletion of exocyst function and subsequent autophagic deficiency contribute added complexity to the cells ability to deal with stress. Progression to TWEAK-Fn14-mediated non-canonical NF-KB activation is intriguing because TWEAK can induce several cell death pathways including caspase-mediated death (Chicheportiche et al, 1997; Nakayama et al, 2003). However, it is important to state that these data are not suggesting Fn14-mediated non-canonical NF-KB is singularly dependent on the effects of autophagic deficiency, nor that this is the sole driving factor behind Exoc5 CKO ureter cell death, rather these data indicate a significant role for impaired autophagic flux in promoting a sustained TWEAK and Fn14 environment.

In summary, these data demonstrate that urothelial cell death is initiated independent of aberrant urothelial stratification and is the underlying pathological driver of congenital obstructive uropathy in the Exoc5 CKO mouse model. We further implicate the effects of impaired autophagy in responding to stress events during ureter development, whereby there is progressive promotion of canonical and then non-canonical NF-KB signaling. These findings may provide insight into the pathological events contributing to human COU.

\section{Methods}

Animals. All animal procedures and protocols were conducted in accordance with IACUC specifications approved by the University of Hawai'i Animal and Veterinary Services. Dr. 
Fogelgren's IACUC approved protocol is \#11-1094. and the University of Hawaii has an Animal Welfare Assurance on file with the Office of Laboratory Animal Welfare (OLAW), assurance number is A3423-01. Mice were housed under standard conditions with a 12-hour light cycle with water and food ad libitum. The floxed EXOC5 mouse strain (EXOC5 $\left.{ }^{\mathrm{FL}}\right)$ was generated and used as previously described in Fogelgren, et. al, 2015, as was the tdTomato Cre reporter strain (Jackson Laboratories stock \#007909). The Ksp-Cre and UPK3a-GCE mouse strains were obtained from Jackson Laboratories (stock \# 012237 and 015855, respectively). All mice were on a C57/BI6/J inbred background. For timed matings, females mated with a male were monitored for abdominal bulging beginning fourteen days after arranging timed mating. Embryos were dissected in the morning and measured by embryonic body length and staged using Theiler staging criteria (TS) to ensure the developmental stage of each embryo. 6-8 week old UPK3aGCE mice were fed tamoxifen-containing chow (Cat \#TD.130860, ENVIGO) for two weeks, and then after four weeks with normal chow, bladders were collected for histology analysis. For rescue experiments, timed mated female mice were i.p. injected at E16.5 with z-VAD-fmk (R\&D Systems, Cat \#FMK001) at $5 \mu \mathrm{g} / \mathrm{g}$ body weight in 10\% DMSO in PBS. Embryos were collected 48 hours after injection, with caudal torsos collected for histological analysis and DNA isolated for genotyping.

Histology and immunohistochemistry. Caudal torsos of Exoc5 knockout and control animals were dissected and fixed in $4 \%$ formaldehyde overnight with rocking at $4{ }^{\circ} \mathrm{C}$. Samples were embedded in paraffin according to standard protocols and cut into $5 \mu \mathrm{m}$ sections. Staining and immunohistochemistry procedures were performed as previously reported (Fogelgren et al., 2015). Primary antibodies used were: anti-E-cadherin at 1:200 (Cell Signaling, Cat \#3195), antiSmooth Muscle Actin at 1:800 (Millipore Sigma, Cat \#A2547), anti-Uroplakin-3 at 1:100 (ARP, 03610108), and anti-LC3 at 1:500 (Cell Signaling, Cat. \#12741). Secondary antibodies (Dylight, 35552 and 35560$)$ and were used at 1:800 at wavelengths 488 and $594 \mathrm{~nm}$. Stained sections 
were analyzed using a fluorescent Olympus BX41 microscope. LC3 quantification analysis was performed using ImageJ software (NIH).

Co-immunoprecipitations (co-IPs) and western blots. Samples were lysed in coimmunoprecipitation buffer (50nM Tris-HCL (pH 8), 150mM NaCl, 5mM EDTA, 0.5\% NP- 40, 1mM DTT, 20mM NaF) containing phosphatase and protease inhibitors via mechanical homogenizing and vortex. Samples were placed microcentrifuge and spun at $14,000 \mathrm{rpm}$ at $4^{\circ} \mathrm{C}$ for 30 minutes. The supernatant was removed and proteins were quantified by Bradford's assay. Protein input for co-immunoprecipitation was $2 \mathrm{mg}$ and $8 \mu \mathrm{g}$ of antibody was used per $2 \mathrm{mg}$ of protein input. Protein samples were incubated with antibody against EXOC4 (Enzo, ADI-VAM-SV016-D) or rat IgG control (Fisher, Cat \#02-9602) overnight with end-to-end rotation in $4^{\circ} \mathrm{C}$. Immune complexes were then pulled down using Protein A/G Magnetic Beads (Thermo Fisher, Cat \#88802), boiled in 2x Laemmli Sample Buffer (Biorad, Cat \#1610737) with $\beta$-mercaptoethanol. The supernatant was run using SDS-PAGE and transferred onto nitrocellulose membrane using Trans-Blot Turbo Transfer System. The membrane was blocked in 5\% nonfat milk for 1 hour and probed with primary antibody overnight. Secondary antibodies at $600 \mathrm{~nm}$ or $800 \mathrm{~nm}$ (Licor IRDye) were incubated at 1:10,000 for 1 hour followed by 3x PBSt washes and scanned on Odyssey CLx Imaging System.

Other antibodies used in western analysis include p62 (Cell Signaling, Cat \#16177S), ATG7 (Cell Signaling, Cat \#8558), ATG5 (Cell Signaling, Cat \#12994), EXOC5 (SEC10) (Santa Cruz, Cat \#sc-514802), LC3 (Cell Signaling, Cat \#12741), Fn14 (Abcam, Cat \#EPR3179). ImageStudioLite (LiCor) was used to analyze and pseudocolor all scans to grayscale.

Cell culture. For immunofluorescence, cells were seeded on coverslips and grown overnight.

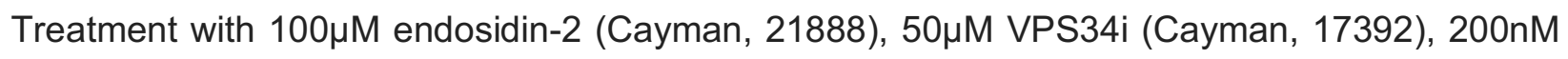
BafA1 (Sigma-Aldrich, B1793) were applied in fresh media change at implicated time and grown under standard conditions at $37^{\circ} \mathrm{C}$ and $5 \% \mathrm{CO}_{2}$ for the respective timepoints listed. Cells were washed three times with PBS followed by fixation for 10 minutes in $4 \%$ PFA. Cells were 
permeabilized for 10 minutes with $0.1 \%$ Triton $\mathrm{X}-100$ with $3 x$ PBSt washes occurring between each step. Cells were blocked in 5\% BSA in PBSt for 1 hour then incubated with the respective primary antibody $(1: 100)$ overnight at $4^{\circ} \mathrm{C}$. Primary antibodies used were EXOC5 (SEC10), ATG5, p65 (Cell Signaling, Cat\# 8242S, p52 (Millipore, Cał\# 05-361), GOL97 (Santa Cruz, Cat \#sc-59820), Fn14. Cells were washed 3x PBSt then incubated with secondary antibody (Dylight) for 1 hour, washed with $3 x$ PBSt for 5 minutes and mounted using VECTASHIELD® Antifade Mounting Medium or PBS, and sealed with nail polish.

Crystal violet staining. 300k SV-HUC-1 cells were seeded and transfected with $3 \mu \mathrm{g}$ MISSON pLKO.1-puro non-mammalian targeting control shRNA (Sigma, Cat\# SHC002) or shExoc5 (Sigma, Cat\# TRCN0000061963) using RNAiMAX (Thermo Fisher, Cat \#13778075,) according to the manufacturer's protocol. Transfected cells were grown for 72 hours followed by puromycin selection. Stable cell lines were confirmed for EXOC5 knockdown by western and plated at 100k cells per well in 6 well plates in triplicate and grown for 1 week under vehicle control or 200nM BafA1 followed by staining with $0.1 \%$ crystal violet (CV) solution. CV solution was removed with repeated $\mathrm{H}_{2} \mathrm{O}$ washes and the plate was scanned. Percent area coverage was measured using ImageJ software and set relative to control.

Cell Viability Assay. 10K SV-HUC-1 cells were seeded per well of a 96-well opaque plate. Cells were treated and then subsequently assessed for their cell viability by the CellTiter-Glo Luminescent Cell Viability Assay (Promega, Cat \#G7570). The assay measures ATP as a biomarker of metabolically active cells by the luminescence released from the conversion of Beetle Luciferin + ATP with the enzyme catalyst Ultra-Glo recombinant luciferase and $\mathrm{Mg}_{2+}$ to oxyluciferin + AMP + PPi + CO2 and light. $50 \mu \mathrm{L}$ of CellTiterGlo reagent and buffer mix was added to $50 \mu \mathrm{L}$ of cell media in each well and luminescence was measured by SpectraMax M3 (Molecular Devices,).

Ex vivo ureter culture model. Timed matings were set up and embryos were collected at gestational age 15.5 (E15.5). Ureters were microdissected and placed on $1.0 \mu \mathrm{m}$ sterile filters at 
the air-liquid interface in a 12 well plate in 50/50 DMEM/HAMS F12 media supplemented with $5 \mu \mathrm{g} / \mathrm{ml}$ transferrin, $100 \mu \mathrm{g} / \mathrm{ml}$ penicillin, $100 \mathrm{U} / \mathrm{ml}$ streptomycin. The ureters were cultured for 72 hours at $37^{\circ} \mathrm{C}$. Gross images and peristalsis videos were taken using an Olympus CKX41 microscope. For samples that were treated with chemical agonists or antagonists, media containing the compounds were changed daily; and samples were cultured for 72 hours at $37^{\circ} \mathrm{C}$. After 72 hours in culture, the ureter explants were fixed with $4 \%$ paraformaldehyde overnight and subsequently placed in sucrose for cryo-sectioning and histological analysis. Alternatively, the explants were collected for RNA extraction.

Affymetrix Clariom D GeneChip microarrays. Ureters were microdissected from E16.5 embryos ( $\mathrm{n}=3$ Exoc5 $5^{F L / F L}$ control and $\mathrm{n}=3$ Exoc5 $5^{F L F L} ;$ Cre $^{K s p}$ CKOs) and RNA was isolated as previous described (Lee et al., 2016). After confirming RNA quality with an Agilent Bioanalyzer, gene expression profiling was performed at the University of Hawaii Genomics and Bioinformatics Shared Resources using Affymetrix Clariom D gene chips. Transcriptome analysis software (TAC, Affymetrix) was used to analyze and identify differential expression between wild type and Exoc5CKO samples. GraphPad Prism 9 was used to generate a volcano plot and KEGG analysis was performed with the Database for Annotation, Visualization and Integrated Discovery (DAVID) (Huang et al, 2009; Sherman et al, 2008).

Statistical analysis. All experiments were performed at least twice in triplicate with the most representative images shown. Error bars are presented as means \pm SD and differences between groups were analyzed by two-tailed Student's $t$ test or one-way (ANOVA) where listed respectfully. Statistical significance was accepted at $p^{*} \leq 0.05, p^{* *} \leq 0.01 p^{* *} \leq 0.001$ $p^{* * * *} \leq 0.0001$ 


\section{ACKNOWLEDGMENTS}

This work was supported by grants from the National Institutes of Health [grant numbers R01DK117308, R03DK100738, P20GM103456-06A1-8293 to B.F.]; and the March of Dimes [Basil O'Connor Starter Scholar Research Award, grant number \#5-FY14-56 to B.F.]. We thank Tina Carvalho at the University of Hawaii Biological Electron Microscope Facility for her expertise in electron microscopy, the Histopathology Core (supported by NIH G12MD007601, P30GM103341, and U54MD007601) for outstanding histology services, and the Genomics and Bioinformatics Shared Resources for gene profiling support (P30CA071789). 


\section{References}

Ahmed SM, Nishida-Fukuda H, Li Y, McDonald WH, Gradinaru CC, Macara IG (2018) Exocyst dynamics during vesicle tethering and fusion. Nature Communications 9: 5140

Bechtel W, Helmstädter M, Balica J, Hartleben B, Kiefer B, Hrnjic F, Schell C, Kretz O, Liu S, Geist F et al (2013) Vps34 deficiency reveals the importance of endocytosis for podocyte homeostasis. Journal of the American Society of Nephrology : JASN 24: 727-743

Bodemann BO, Orvedahl A, Cheng T, Ram RR, Ou Y-H, Formstecher E, Maiti M, Hazelett CC, Wauson EM, Balakireva M et al (2011) RalB and the Exocyst Mediate the Cellular Starvation Response by Direct Activation of Autophagosome Assembly. Cell (Cambridge) 144: $253-267$

Chevalier RL, Thornhill BA, Forbes MS, Kiley SC (2010) Mechanisms of renal injury and progression of renal disease in congenital obstructive nephropathy. Pediatr Nephrol 25: 687-697

Chicheportiche Y, Bourdon PR, Xu H, Hsu Y-M, Scott H, Hession C, Garcia I, Browning JL (1997) TWEAK, a New Secreted Ligand in the Tumor Necrosis Factor Family That Weakly Induces Apoptosis. The Journal of biological chemistry 272: 32401-32410

Chien Y, Kim S, Bumeister R, Loo Y-M, Kwon SW, Johnson CL, Balakireva MG, Romeo Y, Kopelovich L, Gale M et al (2006) RalB GTPase-Mediated Activation of the IkB Family Kinase TBK1 Couples Innate Immune Signaling to Tumor Cell Survival. Cell (Cambridge) 127: $157-170$

Chua A, Cramer C, Moudgil A, Martz K, Smith J, Blydt-Hansen T, Neu A, Dharnidharka VR (2019) Kidney transplant practice patterns and outcome benchmarks over 30 years: The 2018 report of the NAPRTCS. Pediatric transplantation 23: e13597-n/a

Coulter ME, Musaev D, DeGennaro EM, Zhang X, Henke K, James KN, Smith RS, Hill RS, Partlow JN, Muna A-S et al (2020) Regulation of human cerebral cortical development by EXOC7 and EXOC8, components of the exocyst complex, and roles in neural progenitor cell proliferation and survival. Genetics in Medicine 22: 1040-1050

Craven A-MS, Hawley CM, McDonald SP, Rosman JB, Brown FG, Johnson DW (2007) PREDICTORS OF RENAL RECOVERY IN AUSTRALIAN AND NEW ZEALAND ENDSTAGE RENAL FAILURE PATIENTS TREATED WITH PERITONEAL DIALYSIS. Peritoneal dialysis international 27: 184-191

Fogelgren B, Polgar N, Lui VH, Lee AJ, Tamashiro KK, Napoli JA, Walton CB, Zuo X, Lipschutz JH (2015) Urothelial Defects from Targeted Inactivation of Exocyst Sec10 in Mice Cause Ureteropelvic Junction Obstructions. PLoS One 10: e0129346

Gandhi D, Molotkov A, Batourina E, Schneider K, Dan H, Reiley M, Laufer E, Metzger D, Liang $\mathrm{F}$, Liao $\mathrm{Y}$ et al (2013) Retinoid signaling in progenitors controls specification and regeneration of the urothelium. Developmental cell 26: 469-482

Goodall Megan L, Fitzwalter Brent E, Zahedi S, Wu M, Rodriguez D, Mulcahy-Levy Jean M, Green Douglas R, Morgan M, Cramer Scott D, Thorburn A (2016) The Autophagy Machinery Controls Cell Death Switching between Apoptosis and Necroptosis. Developmental Cell 37: 337-349

Gump JM, Staskiewicz L, Morgan MJ, Bamberg A, Riches DWH, Thorburn A (2014) Autophagy variation within a cell population determines cell fate through selective degradation of Fap1. Nature cell biology 16: 47-54

Gurunathan S, Winkles JA, Ghosh S, Ghosh S, Hayden MS (2014) Regulation of fibroblast growth factor-inducible 14 (Fn14) expression levels via ligand-independent lysosomal degradation. J biol Chem 289 
Hartleben B, Gödel M, Meyer - Schwesinger C, Liu S, Ulrich T, Köbler S, Wiech T, Grahammer F, Arnold SJ, Lindenmeyer MT et al (2010) Autophagy influences glomerular disease susceptibility and maintains podocyte homeostasis in aging mice.

Heider MR, Gu M, Duffy CM, Mirza AM, Marcotte LL, Walls AC, Farrall N, Hakhverdyan Z, Field MC, Rout MP et al (2016) Subunit connectivity, assembly determinants and architecture of the yeast exocyst complex. Nature structural \& molecular biology 23: 59-66

Honeycutt J, Hammam O, Hsieh MH (2015) Schistosoma haematobium egg-induced bladder urothelial abnormalities dependent on p53 are modulated by host sex. Experimental parasitology 158: 55-60

Huang DW, Sherman BT, Lempicki RA (2009) Bioinformatics enrichment tools: paths toward the comprehensive functional analysis of large gene lists. Nucleic acids research 37: 1-13

Ikner A, Ashkenazi A (2011) TWEAK induces apoptosis through a death-signaling complex comprising receptor-interacting protein 1 (RIP1), Fas-associated death domain (FADD), and caspase-8. The Journal of biological chemistry 286: 21546-21554

Jackson AR, Ching CB, McHugh KM, Becknell B (2020) Roles for urothelium in normal and aberrant urinary tract development. Nature reviews Urology 17: 459-468

Jin J, Xiao Y, Chang J-H, Yu J, Hu H, Starr R, Brittain GC, Chang M, Cheng X, Sun S-C (2012) The kinase TBK1 controls IgA class switching by negatively regulating noncanonical NFKB signaling. Nature immunology 13: 1101-1109

Johansen KL, Chertow GM, Foley RN, Gilbertson DT, Herzog CA, Ishani A, Israni AK, Ku E, Kurella Tamura M, Li S et al (2021) US Renal Data System 2020 Annual Data Report: Epidemiology of Kidney Disease in the United States. American journal of kidney diseases 77: A7-A8

Johnston Amelia J, Murphy Kate T, Jenkinson L, Laine D, Emmrich K, Faou P, Weston R, Jayatilleke Krishnath M, Schloegel J, Talbo G et al (2015) Targeting of Fn14 Prevents Cancer-Induced Cachexia and Prolongs Survival. Cell (Cambridge) 162: 1365-1378

Kim SI, Na H-J, Ding Y, Wang Z, Lee SJ, Choi ME (2012) Autophagy Promotes Intracellular Degradation of Type I Collagen Induced by Transforming Growth Factor (TGF)- $\beta 1$. The Journal of biological chemistry 287: 11677-11688

Komatsu M, Waguri S, Ueno T, Iwata J, Murata S, Tanida I, Ezaki J, Mizushima N, Ohsumi Y, Uchiyama $Y$ et al (2005) Impairment of starvation-induced and constitutive autophagy in Atg7-deficient mice. The Journal of cell biology 169: 425-434

Kroemer G, Mariño G, Levine B (2010) Autophagy and the Integrated Stress Response. Molecular cell 40: 280-293

Kuma A, Hatano M, Matsui M, Yamamoto A, Nakaya H, Yoshimori T, Ohsumi Y, Tokuhisa T, Mizushima N (2004) The role of autophagy during the early neonatal starvation period. Nature 432: 1032-1036

Lee AJ, Polgar N, Napoli JA, Lui VH, Tamashiro KK, Fujimoto BA, Thompson KS, Fogelgren B (2016) Fibroproliferative response to urothelial failure obliterates the ureter lumen in a mouse model of prenatal congenital obstructive nephropathy. Sci Rep 6: 31137

Martin TD, Chen X-W, Kaplan REW, Saltiel AR, Walker CL, Reiner DJ, Der CJ (2014) Ral and Rheb GTPase activating proteins integrate mTOR and GTPase signaling in aging, autophagy, and tumor cell invasion. Molecular cell 53: 209-220

Martin-Sanchez D, Fontecha-Barriuso M, Carrasco S, Sanchez-Niño MD, Mässenhausen Av, Linkermann A, Cannata-Ortiz P, Ruiz-Ortega M, Egido J, Ortiz A et al (2018) TWEAK and RIPK1 mediate a second wave of cell death during AKI. Proceedings of the National Academy of Sciences 115: 4182

Mathew R, Karp CM, Beaudoin B, Vuong N, Chen G, Chen H-Y, Bray K, Reddy A, Bhanot G, Gelinas C et al (2009) Autophagy Suppresses Tumorigenesis through Elimination of p62. Cell (Cambridge) 137: 1062-1075 
Mei K, Li Y, Wang S, Shao G, Wang J, Ding Y, Luo G, Yue P, Liu J-J, Wang X et al (2018) CryoEM structure of the exocyst complex. Nature Structural \& Molecular Biology 25: 139-146

Meng Q, Cai D (2011) Defective hypothalamic autophagy directs the central pathogenesis of obesity via the IkappaB kinase beta (IKKbeta)/NF-kappaB pathway. The Journal of biological chemistry 286: 32324-32332

Mesrobian HG, Mirza SP (2012) Hydronephrosis: a view from the inside. Pediatr Clin North Am 59: 839-851

Miklovicova D, Cervenova O, Cernianska A, Jancovicova Z, Dedik L, Vasilenkova A (2008) Longterm follow-up of renal function in patients after surgery for obstructive uropathy. Pediatric nephrology (Berlin, West) 23: 937-945

Mizushima N, Levine B (2010) Autophagy in mammalian development and differentiation. Nature Cell Biology 12: 823-830

Mohseni N, McMillan SC, Chaudhary R, Mok J, Reed BH (2009) Autophagy promotes caspasedependent cell death during Drosophila development. Autophagy 5: 329-338

Morin A, Cordelieres FP, Cherfils J, Olofsson B (2010) RhoGDI3 and RhoG: Vesicular trafficking and interactions with the Sec3 Exocyst subunit. Small Gtpases 1: 142-156

Moskalenko S, Henry DO, Rosse C, Mirey G, Camonis JH, White MA (2002) The exocyst is a Ral effector complex. Nature Cell Biology 4: 66-72

Muñoz-García B, Martín-Ventura JL, Martínez E, Sánchez S, Hernández G, Ortega L, Ortiz A, Egido J, Blanco-Colio LM (2006) Fn14 Is Upregulated in Cytokine-Stimulated Vascular Smooth Muscle Cells and Is Expressed in Human Carotid Atherosclerotic Plaques. Stroke 37: 2044-2053

Nagy D, Ennis KA, Wei R, Su SC, Hinckley CA, Gu R-F, Gao B, Massol RH, Ehrenfels C, Jandreski $L$ et al (2021) Developmental synaptic regulator, TWEAK/Fn14 signaling, is a determinant of synaptic function in models of stroke and neurodegeneration. Proceedings of the National Academy of Sciences 118: e2001679118

Nakayama M, Ishidoh K, Kojima Y, Harada N, Kominami E, Okumura K, Yagita H (2003) Fibroblast growth factor-inducible 14 mediates multiple pathways of TWEAK-induced cell death. Journal of immunology (Baltimore, Md : 1950) 170: 341-348

Nezis IP, Shravage BV, Sagona AP, Johansen T, Baehrecke EH, Stenmark H (2010) Autophagy as a trigger for cell death: Autophagic degradation of inhibitor of apoptosis dBruce controls DNA fragmentation during late oogenesis in Drosophila. Autophagy 6: 1214-1215

Peng L, Li Q, Wang H, Wu J, Li C, Liu Y, Liu J, Xia L, Xia Y (2018) Fn14 deficiency ameliorates psoriasis-like skin disease in a murine model. Cell death \& disease 9: 801-801

Roth KS, Koo HP, Spottswood SE, Chan JC (2002) Obstructive Uropathy: An Important Cause of Chronic Renal Failure in Children. Clinical Pediatrics 41: 309-314

Runwal G, Stamatakou E, Siddiqi FH, Puri C, Zhu Y, Rubinsztein DC (2019) LC3-positive structures are prominent in autophagy-deficient cells. Scientific Reports 9: 10147

Saitoh T, Nakayama MN, H. Yagita, H., Yamamoto N, Yamaoka S (2003) TWEAK induces NFkappaB2 p100 processing and long lasting NF-kappaB activation. Journal of Biological Chemistry 278: 36005-36012

Sherman BT, Lempicki RA, Huang DW (2008) Systematic and integrative analysis of large gene lists using DAVID bioinformatics resources. Nature protocols 4: 44-57

Shipitsin M, Feig LA (2004) RalA but not RalB enhances polarized delivery of membrane proteins to the basolateral surface of epithelial cells. Molecular and cellular biology 24: $5746-5756$

Simicek M, Lievens S, Laga M, Guzenko D, Aushev VN, Kalev P, Baietti MF, Strelkov SV, Gevaert $\mathrm{K}$, Tavernier J et al (2013) The deubiquitylase USP33 discriminates between RALB functions in autophagy and innate immune response. Nature cell biology 15: 1220-1230

Singh S, Kumari R, Chinchwadkar S, Aher A, Matheshwaran S, Manjithaya R (2019) Exocyst Subcomplex Functions in Autophagosome Biogenesis by Regulating Atg9 Trafficking. Journal of molecular biology 431: 2821-2834 
Takahashi A, Kimura T, Takabatake Y, Namba T, Kaimori J, Kitamura H, Matsui I, Niimura F, Matsusaka T, Fujita N et al (2012) Autophagy Guards Against Cisplatin-Induced Acute Kidney Injury. The American journal of pathology 180: 517-525

Tang C, Livingston MJ, Liu Z, Dong Z (2020) Autophagy in kidney homeostasis and disease. Nature reviews Nephrology 16: 489-508

TerBush DR, Maurice T, Roth D, Novick P (1996) The Exocyst is a multiprotein complex required for exocytosis in Saccharomyces cerevisiae. The EMBO journal 15: 6483-6494

Thorburn J, Andrysik Z, Staskiewicz L, Gump J, Maycotte P, Oberst A, Green DR, Espinosa JM, Thorburn A (2014) Autophagy controls the kinetics and extent of mitochondrial apoptosis by regulating PUMA levels. Cell reports (Cambridge) 7: 45-52

Tracy K, Velentzas PD, Baehrecke EH (2016) Ral GTPase and the exocyst regulate autophagy in a tissue-specific manner. EMBO reports 17: 110-121

Truschel ST, Clayton DR, Beckel JM, Yabes JG, Yao Y, Wolf-Johnston A, Birder LA, Apodaca G (2018) Age-related endolysosome dysfunction in the rat urothelium. PLOS ONE 13: e0198817

Uhm M, Bazuine M, Zhao P, Chiang S-H, Xiong T, Karunanithi S, Chang L, Saltiel AR (2017) Phosphorylation of the exocyst protein Ex084 by TBK1 promotes insulin-stimulated GLUT4 trafficking. Science Signaling 10: eaah5085

Unudurthi SD, Nassal DM, Patel NJ, Thomas E, Yu J, Pierson CG, Bansal SS, Mohler PJ, Hund TJ (2020) Fibroblast growth factor-inducible 14 mediates macrophage infiltration in heart to promote pressure overload-induced cardiac dysfunction. Life Sciences 247: 117440

Van Bergen NJ, Ahmed SM, Collins F, Cowley M, Vetro A, Dale RC, Hock DH, de Caestecker C, Menezes M, Massey S et al (2020) Mutations in the exocyst component EXOC2 cause severe defects in human brain development. The Journal of experimental medicine 217

Verbitsky M, Westland R, Perez A, Kiryluk K, Liu Q, Krithivasan P, Mitrotti A, Fasel DA, Batourina E, Sampson MG et al (2019) The copy number variation landscape of congenital anomalies of the kidney and urinary tract. Nature Genetics 51: 117-127

Vince JE, Chau D, Callus B, Wong WW-L, Hawkins CJ, Schneider P, McKinlay M, Benetatos CA, Condon SM, Chunduru SK et al (2008) TWEAK-FN14 signaling induces lysosomal degradation of a cIAP1-TRAF2 complex to sensitize tumor cells to TNFalpha. The Journal of cell biology 182: 171-184

Wooten MW, Geetha T, Seibenhener ML, Babu JR, Diaz-Meco MT, Moscat J (2005) The p62 scaffold regulates nerve growth factor-induced NF-kappaB activation by influencing TRAF6 polyubiquitination. The Journal of biological chemistry 280: 35625-35629

Xiao G, Harhaj E, Sun S-C (2001) NF- Kappa B-Inducing Kinase Regulates the Processing of NF- Kappa B2 p100. Molecular Cell 7: 401-409

Zhang C, Brown MQ, van de Ven W, Zhang Z-M, Wu B, Young MC, Synek L, Borchardt D, Harrison R, Pan S et al (2016) Endosidin2 targets conserved exocyst complex subunit EXO70 to inhibit exocytosis. Proceedings of the National Academy of Sciences - PNAS 113: E41-E50

Zhao Z, Burkly LC, Campbell S, Schwartz N, Molano A, Choudhury A, Eisenberg RA, Michaelson JS, Putterman C (2007) TWEAK/Fn14 Interactions Are Instrumental in the Pathogenesis of Nephritis in the Chronic Graft-versus-Host Model of Systemic Lupus erythematosus. The Journal of Immunology 179: 7949 
Figure 1. Urothelial cell death occurs independent of failed stratification. (A) Ex vivo ureter explant culture model results in viable Exoc5 $5^{F L F L}$ (control) ureter explants after 72 hours. Growth of E15.5 (t=0 hours) control ureter explants shown after cultured for 72 hours. Explants were fixed, cryosectioned, and immunostained for Ecadherin (green) and SMA (red), which revealed urothelial stratification and strengthening of the smooth muscle layer. Staining for uroplakin-3 (UPK3) (red) demonstrated successful differentiation of superficial cells ex vivo. (B) Time lapse images of ureter explant peristalsis confirmed functional viability of ureters after 72 hours. (C) Wildtype ureter ex vivo treated with retinoic acid inhibitor BMS493 at $100 \mu \mathrm{M}$ for 72 hours developed a monolayered epithelium and lacked uroplakin expression with no apparent cell death, while vehicle control treated ureter explants developed a multilayered urothelium as indicated by E-cadherin (green) and uroplakin-3 (red). (D) Exoc5 CKO ex vivo ureter explants were cultured for $72 \mathrm{~h}$ hours displayed a disrupted urothelial layer with epithelial cells detaching from the smooth muscle layer and entering the lumen. Exoc5 $5^{F L / F L}$ control ex vivo ureter cultures showed differentiation of a multilayered urothelium indicated by E-cadherin (green) and presence of uroplakin-3 (red). Scale bars $=20 \mu \mathrm{M}$.

Figure 2. NF-kB signaling and autophagy are significantly impacted by Exoc5 ablation in developing mouse ureter. (A) Gene expression profiling of total ureter RNA from control and Exoc5 CKO mice at E16.5 ( $n=3$ each group). Volcano plot displaying expression analysis shows uroplakin genes as significantly downregulated (red) and NF-kB signaling mediator, TNFRSF12A (Fn14), as significantly upregulated (green). (B) NF-kB signaling was among the most upregulated pathways identified by KEGG Pathway Analysis. (C) Realtime qPCR confirmed uroplakin-3 downregulation and Fn14 upregulation in Exoc5 CKO E16.5 ureters. In addition, the cytokine TWEAK was significantly upregulated along with it's receptor Fn14. (D) Western blot analysis of Exoc5 CKO ureters showed robust expression of Fn14 at E17.5 when compared to control ureters or even Exoc5 at E16.5. (E) Quantification of Fn14 detected by western blots in E16.5 and E17.5 control and Exoc5 CKO ureters $(n=14,14,11,14$, respectively) shown with each dot representing a mouse. (F) Immunohistochemistry of E17.5 control and Exoc5 CKO ureters demonstrated Fn14 localization to the urothelial cells surrounding the ureter lumen. (G) KEGG Pathway Analysis of the most downregulated pathways indicated broad metabolic disruption. $(\mathrm{H})$ Immunohistochemistry of E17.5 control and Exoc5 CKO ureters displayed 
differences in LC3 distribution and onset of LC3 puncta formation (red, arrow). As expected, uroplakin-3 (green) shows control ureters have stratified by this timepoint but Exoc5 CKO ureters have not. (I) Higher magnification of the accumulation of LC3 in the E17.5 Exoc5 CKO ureter. Statistical significance denoted by n.s.= not significant $\mathrm{p} \geq 0.05, \mathrm{p}^{*} \leq 0.05, \mathrm{p}^{* *} \leq 0.01 \mathrm{p}^{* * *} \leq 0.001$. Scale bars $=20 \mu \mathrm{M}$.

Figure 3. Inhibiting exocyst function in pHUCs results in vesicle accumulation and decreased autophagy. (A) Phase contrast microscopy of $24 \mathrm{~h} 100 \mu \mathrm{M}$ endosidin-2 (ES2) treated pHUC cells display vesicle accumulation. (B) Quantification of control and ES2 treated cells with $>3$ vesicles $(n=854,778)$ show ES2 treated fields more likely to display pHUCs with vesicle accumulation. (C) Immunocytochemistry of $24 \mathrm{~h}$ ES2 treated pHUCs showed the effect on EXOC5 (green) and ATG5 (red) colocalization. (D,E) Western Blot of $24 \mathrm{~h}$ ES2 treated pHUCs indicated increased p62 and LC3 II/I ratio, with a reduction in total ATG5 also observed. (F) In SV-HUC-1 cells, co-immunoprecipitation of EXOC4 successfully pulled down ATG7 denoting a biochemical protein-protein interaction. Statistical significance denoted by $n . s .=$ not significant $p \geq 0.05, p^{*} \leq 0.05, p^{* *} \leq 0.01$ $\mathrm{p}^{* * *} \leq 0.001$. Scale bars $=20 \mu \mathrm{M}$.

Figure 4. Exoc5 ablation in adult bladder urothelial cells results lysosomal accumulation. (A) Specificity of the Cre reporter system shown by tdTomato activation in superficial urothelial cells. (B) Transmission electron microscopy of bladder sections display lysosomal buildup as marked by electron dense organelles. (C) Immunohistochemistry of EXOC5-IUKO bladder sections displayed an accumulation of LC3+ puncta in the superficial urothelial cells. (D) Quantification of LC3+ puncta aggregates per cell with each dot representing a mouse. Statistical significance denoted by n.s. $=$ not significant $p \geq 0.05, p^{*} \leq 0.05, p^{* *} \leq 0.01 p^{* * *} \leq 0.001$. Scale bars $=20 \mu \mathrm{M}$.

Figure 5. Autophagy inhibition induces NF-kB activator, Fn14, response. (A, B) Western Blot analysis of SV-HUC-1 cells treated with $20 \mu \mathrm{M}$ cisplatin for 4 hours to induce DNA damage showed no change in Fn14. (C, D) Western Blot analysis of SV-HUC-1 cells dosed with UV $40 \mathrm{~J} / \mathrm{m}^{2}$ then left to recover for 6 hours showed no increase in Fn14. (E, F) Stably selected shExoc5 SV-HUC-1 cells displayed a synergistic sensitivity in inducing 
Fn14 response when treated with 200nM BafA1 to inhibit autophagy for $24 \mathrm{~h}$ when compared to shCtrl. (G) Western blot quantitation of 200nM BafA1 treatment for 24h increases Fn14 in both control and shExoc5 groups and that this effect is highest in shExoc5 + BafA1 $(\mathrm{H})$ SV-HUC-1 cells grown for a week in the presence of 200nM BafA1 then stained with crystal violet (CV) staining solution showed inhibiting autophagy significantly impaired growth. (I) Quantification of surface area coverage by CV staining. (J) CellTiter-Glo ATP-based cytotoxicity assay of SV-HUC-1 cells treated with BafA1 demonstrated cell death occurring by 72 hours. Statistical significance denoted by n.s. = not significant $p \geq 0.05, p^{*} \leq 0.05, p^{* *} \leq 0.01 p^{* *} \leq 0.001$.

Figure 6. Inhibiting autophagy in SV-HUC-1 cells activates progressive canonical then non-canonical NFkB signaling. (A) Immunocytochemistry of 200nM BafA1 treated SV-HUC-1 cells stained for RelA/p65 (red) showed nuclear translocation as early as $6 \mathrm{~h}$, with sustained localization observed at $24 \mathrm{~h}$ and $48 \mathrm{~h}$. (B) Immunocytochemistry of 200nM BafA1 treated SV-HUC-1 cells demonstrated that nuclear translocation of noncanonical NF-kB constituent p52 (red) was not observed at $6 \mathrm{~h}$ but appeared later at 24h and 48h. (C) TWEAK treated SV-HUC-1 cells stained for RelA/p65 (red) and p52 (red) demonstrated both canonical and noncanonical NF-kB signaling were active at $6 \mathrm{~h}$. (D) Quantification of $6 \mathrm{~h}, 24 \mathrm{~h}$, and $48 \mathrm{~h}$ time course detecting nuclear translocation of RelA/p65 and p52 in response to TWEAK, VPS34i, or BafA1.

\section{Figure 7. Single dose of pan-caspase inhibitor z-VAD-FMK at E16.5 rescues ureter obstruction in Exoc5}

CKO embryos. (A) H\&E staining of E18.5 embryonic ureters from control, Exoc5 CKO with no treatment, and Exoc5 CKO with z-VAD-FMK ureters demonstrate rescue of ureter obstruction and the absence of cell death (Row 1 low mag., Row 2 high mag.). Immunohistochemistry staining of the same series showed Exoc5 CKO mice treated with z-VAD-FMK rescued stratification as indicated by E-cadherin (green) and SMA (red). Positive uroplakin-3 (UPK3) (red) staining in z-VAD-FMK treated Exoc5 CKO indicated successful differentiation of superficial cells. (Row 1 scale bar $=200 \mu \mathrm{m}$, Rows $2-4$ scale bar $=20 \mu \mathrm{m}$ ). (B) H\&E staining of E18.5 kidneys from Exoc5 CKO mice with and without z-VAD-FMK treatment showed subsequent absence of hydronephrosis (scale bar $=200 \mu \mathrm{m})$. 
Expanded View 1. Real-time qPCR confirmed uroplakin-3 (UPK3), uroplakin-1 (UPK1), and PPARY (PPARgamma) downregulation in BMS493 treated ureter explants. Statistical significance denoted by n.s.= not significant $p \geq 0.05, p^{*} \leq 0.05$.

Expanded View 2. (A) Phase contrast microscopy of SV-HUC-1 cells treated with either (A-I) vehicle control, (A-II) $400 \mu \mathrm{M}$ Endosidin-2, (A-III) $50 \mu \mathrm{M}$ VPS34i, or (A-IV) 200 nM BafA1 for 24h. (B) Immunocytochemistry of SV-HUC-1 cells treated with $50 \mu$ M VPS34i for 6h. (B-I,II) Fn14 (red) localization in relation to the Golgi complex as stained by Golgin97 (green). (B-III,IV) Immunohistochemistry of autophagy marker p62 (green) and nuclear translocation of RelA/p65 (red) in response to $50 \mu \mathrm{M}$ VPS34i treatment. Scale bar $=20 \mu \mathrm{M}$ 
bioRxiv preprint doi: https://doi.org/10.1101/2021.07.05.451173; this version posted July 5, 2021. The copyright holder for this preprint (which was not certified by peer review) is the author/funder. All rights reserved. No reuse allowed without permission. 
A

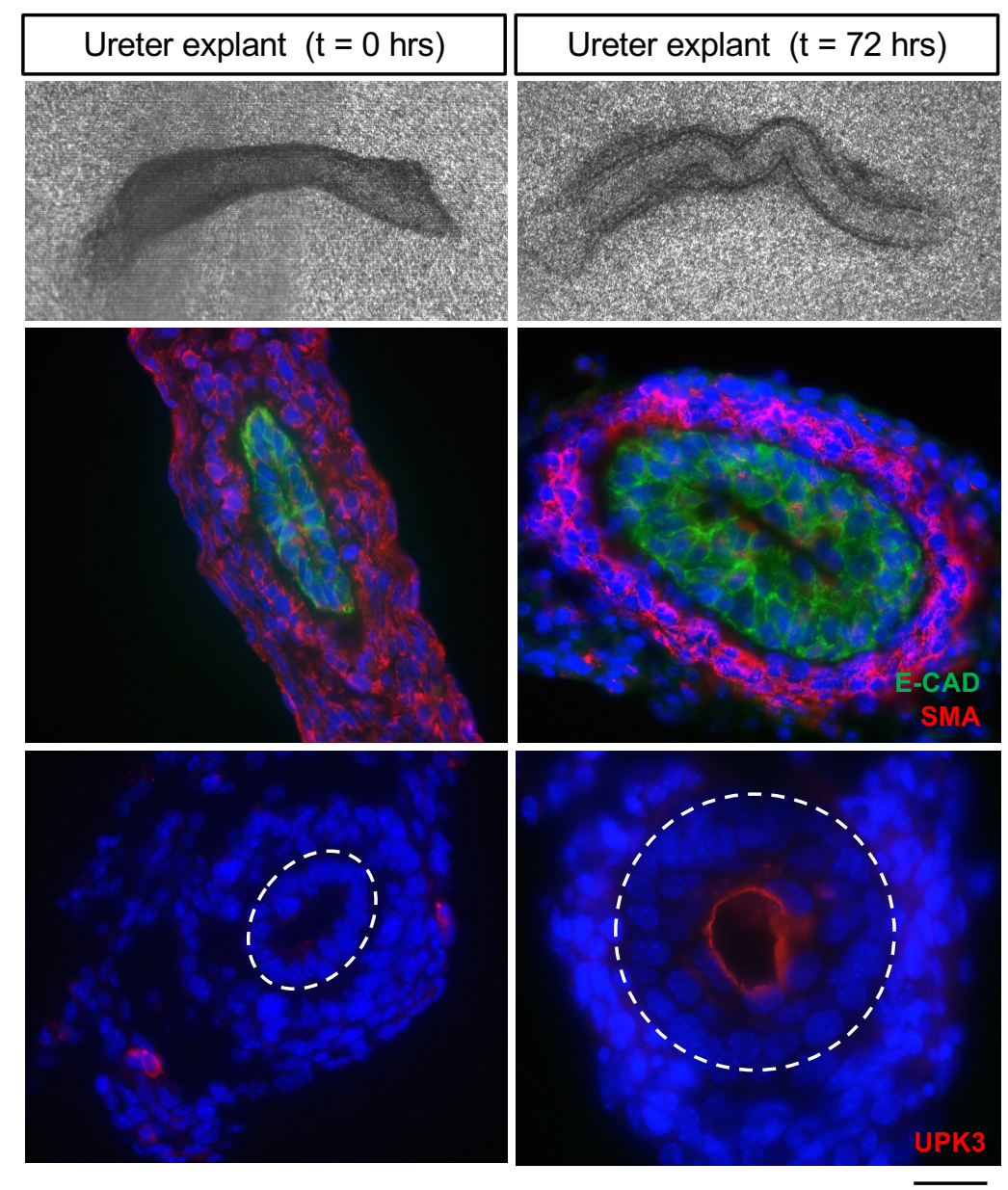

B

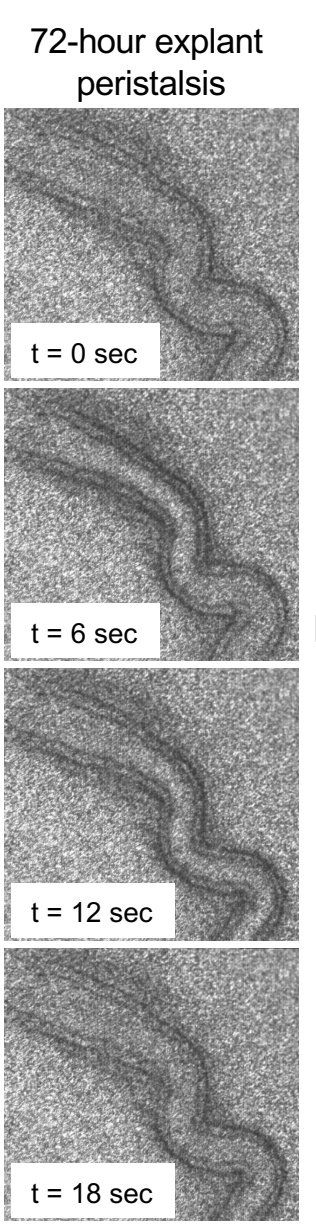

C
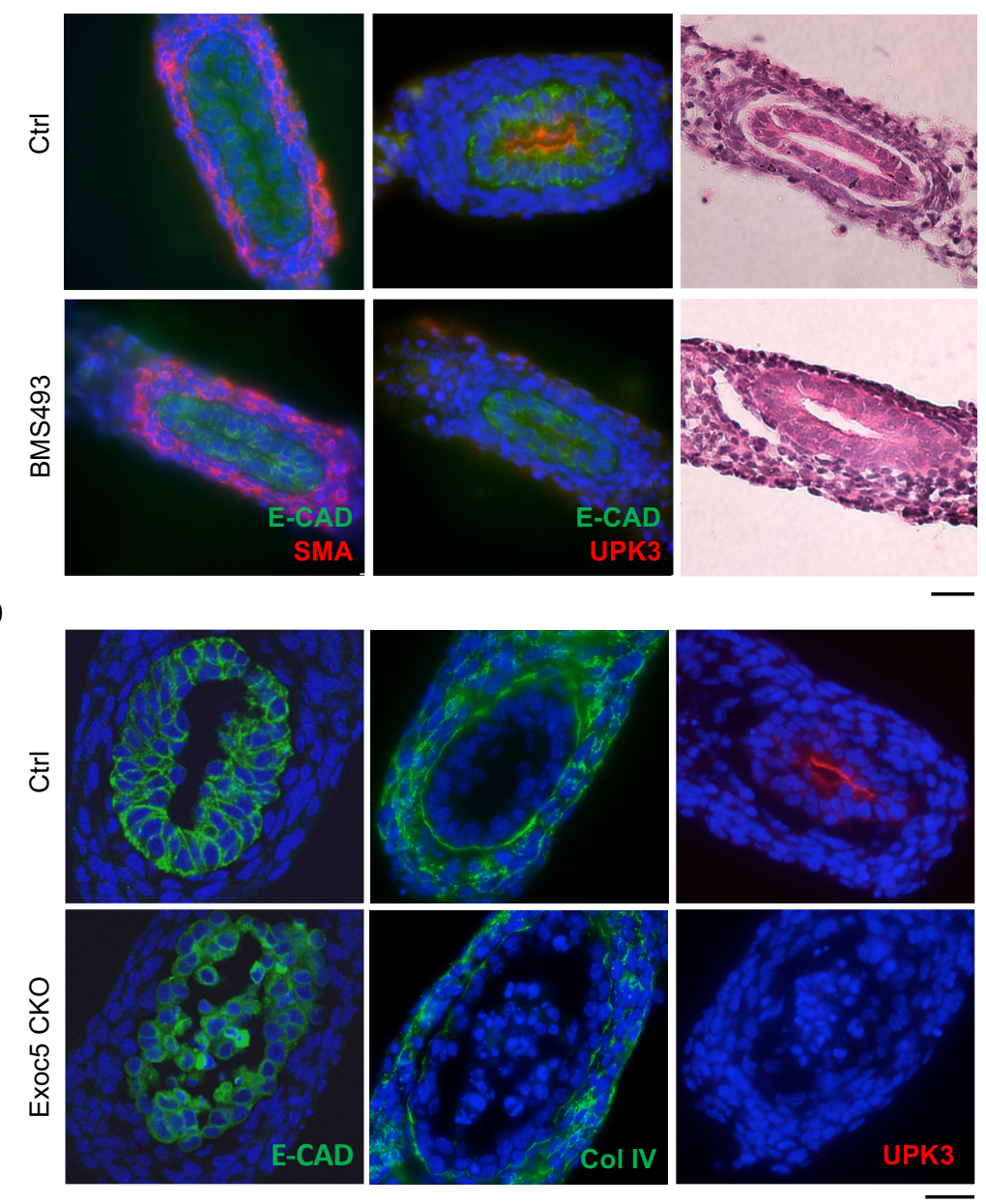

Figure 1 
A

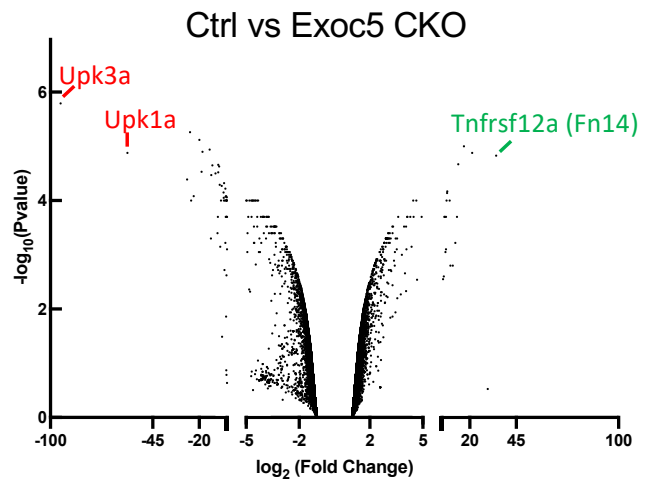

D

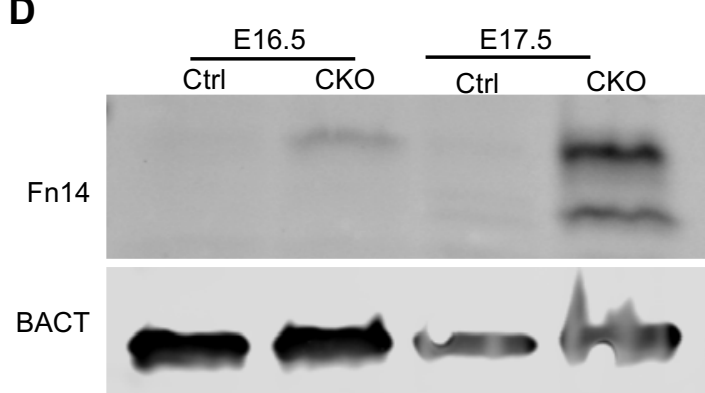

G

KEGG Pathway Analysis (Downregulated)

Metabolism of xenobiotics by cytochrome P450 Drug metabolism - cytochrome P450Chemical Carcinogenesis Glutathione metabolism Metabolic pathways ate interconversions Histidine metabolism Nitrogen metabolism Tyrosine metabolism Retinol metabolism

B

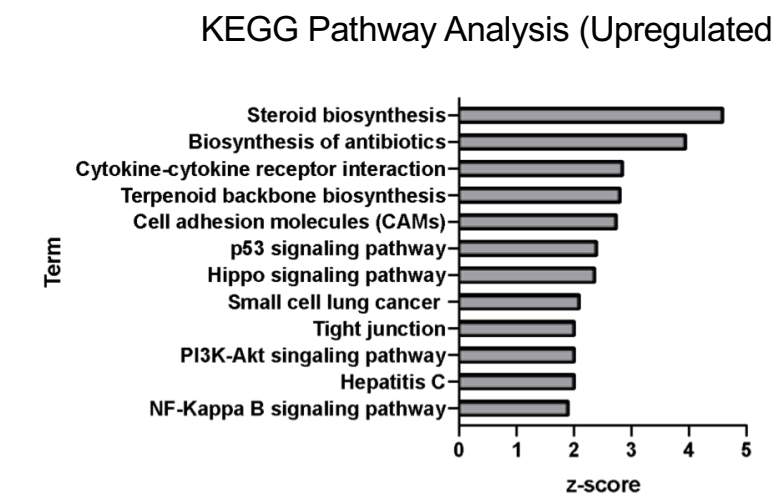

E

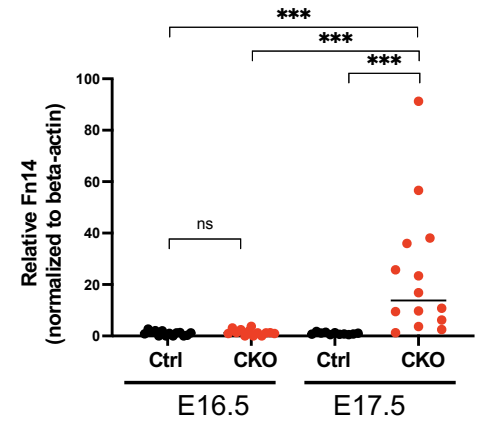

C

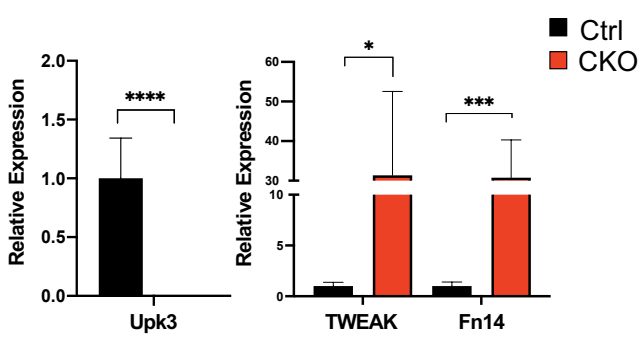

$\mathbf{F}$

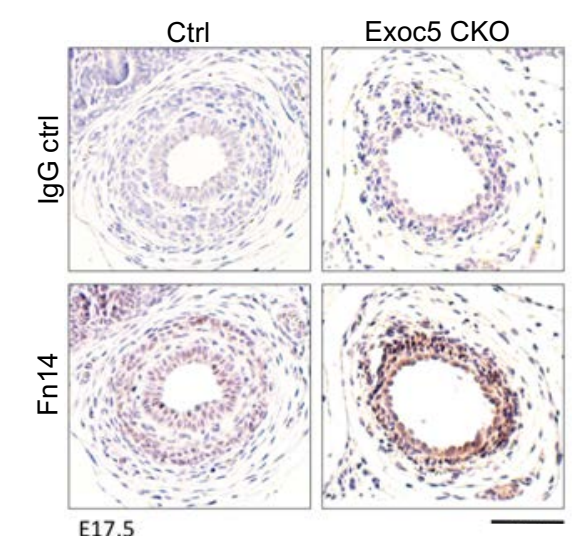

LC3
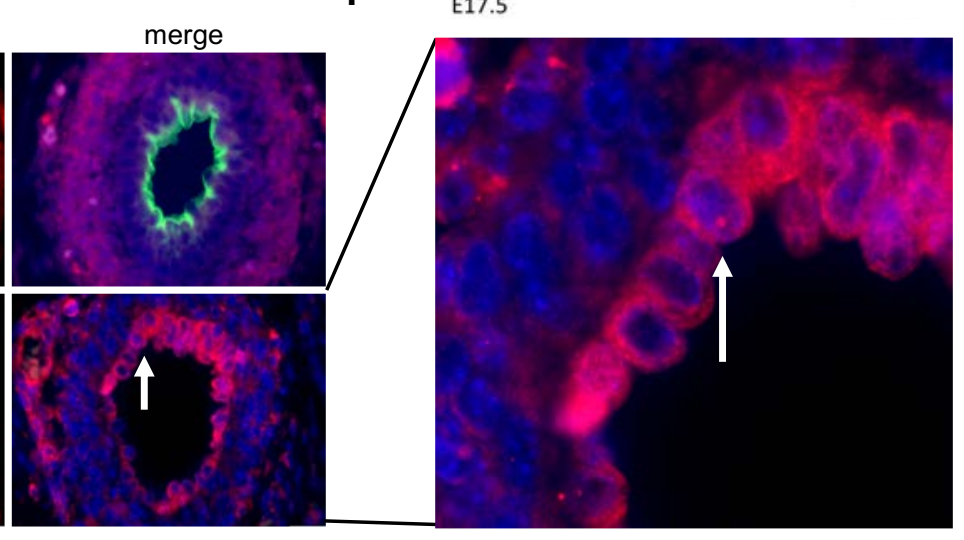

Figure 2 


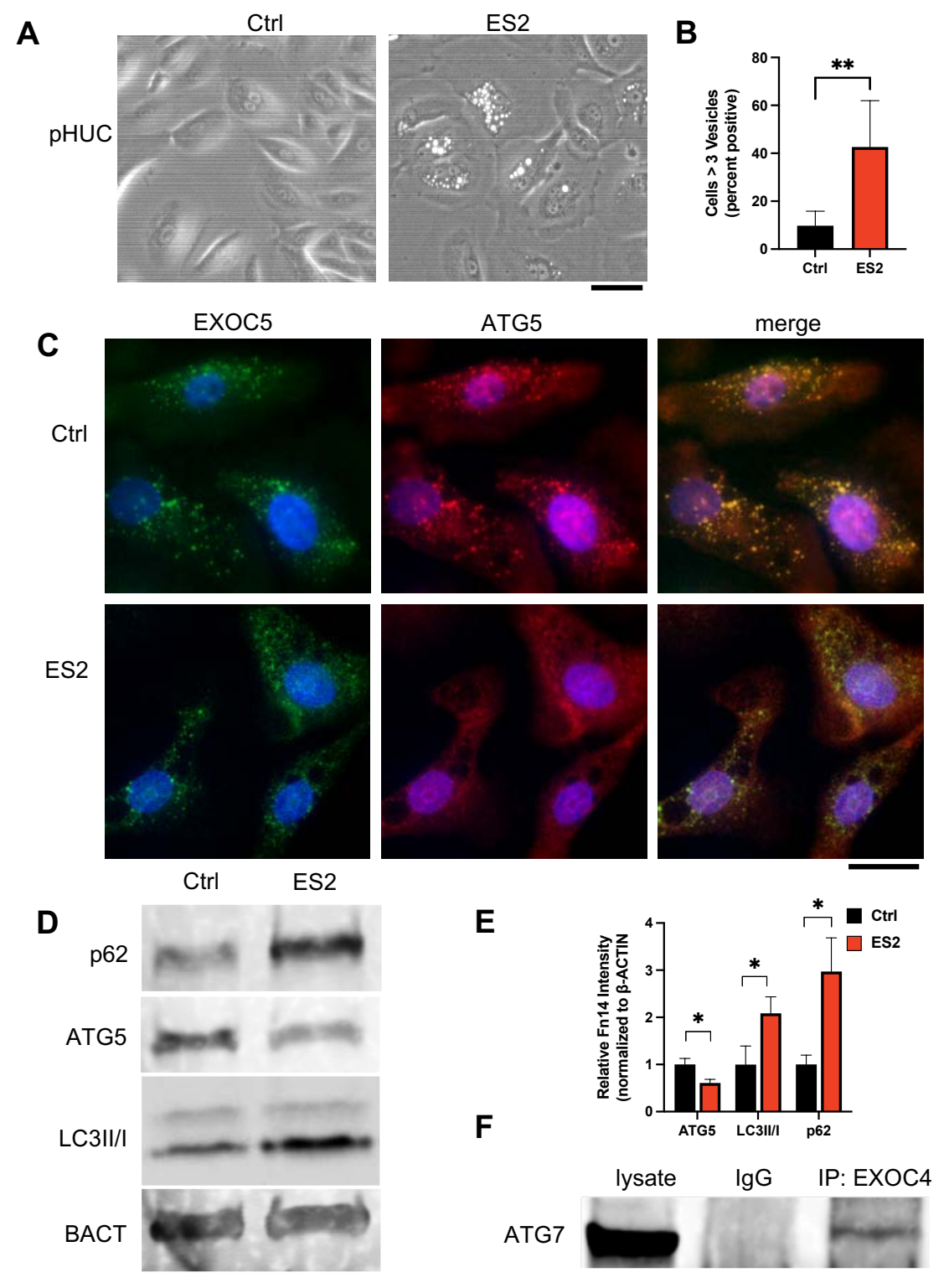

Figure 3 


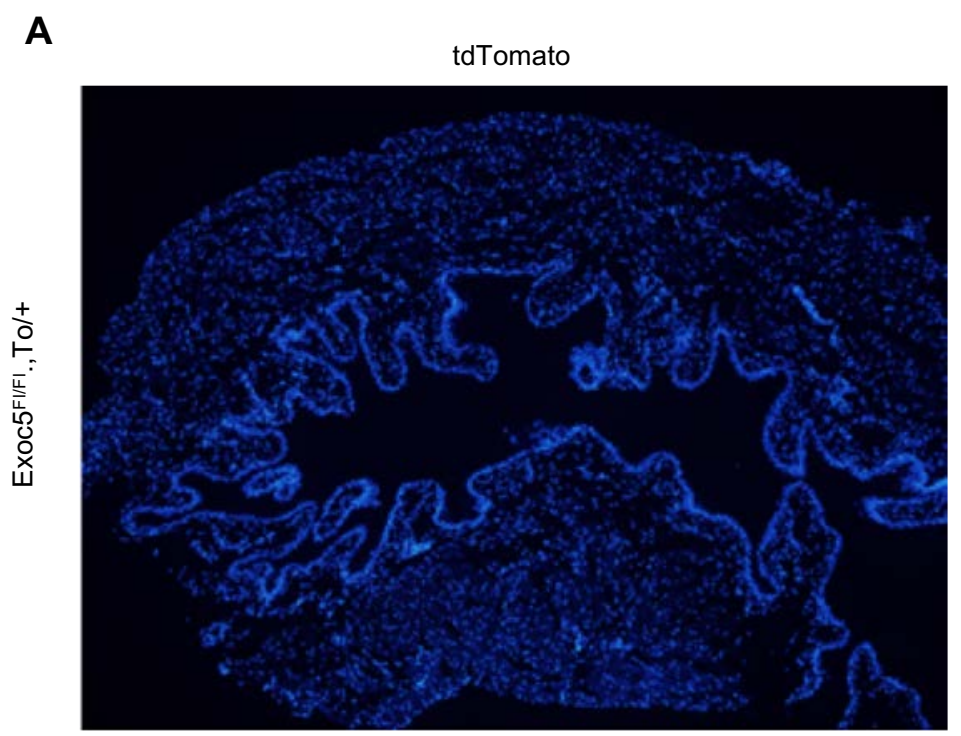

B
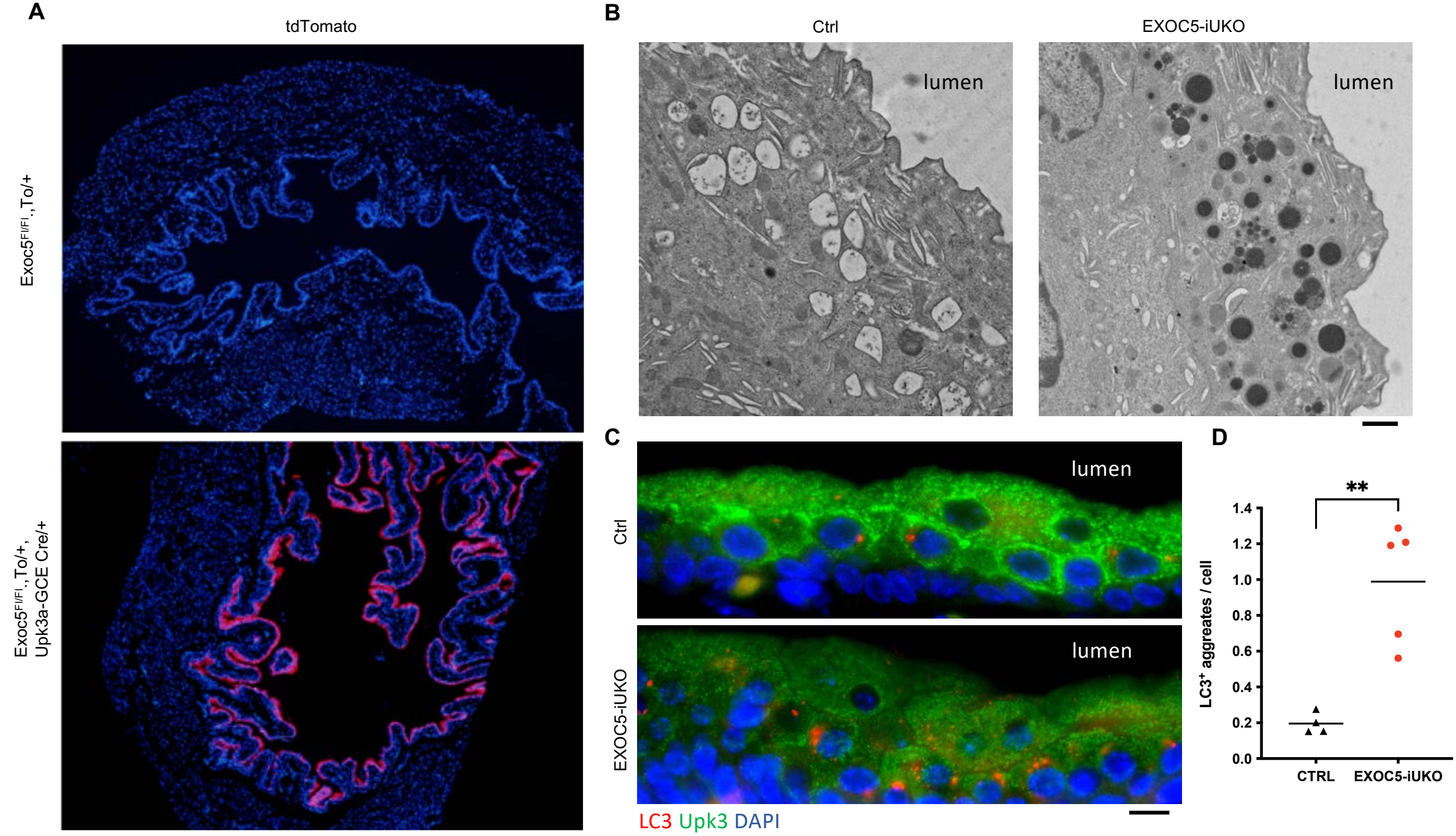

Figure 4 

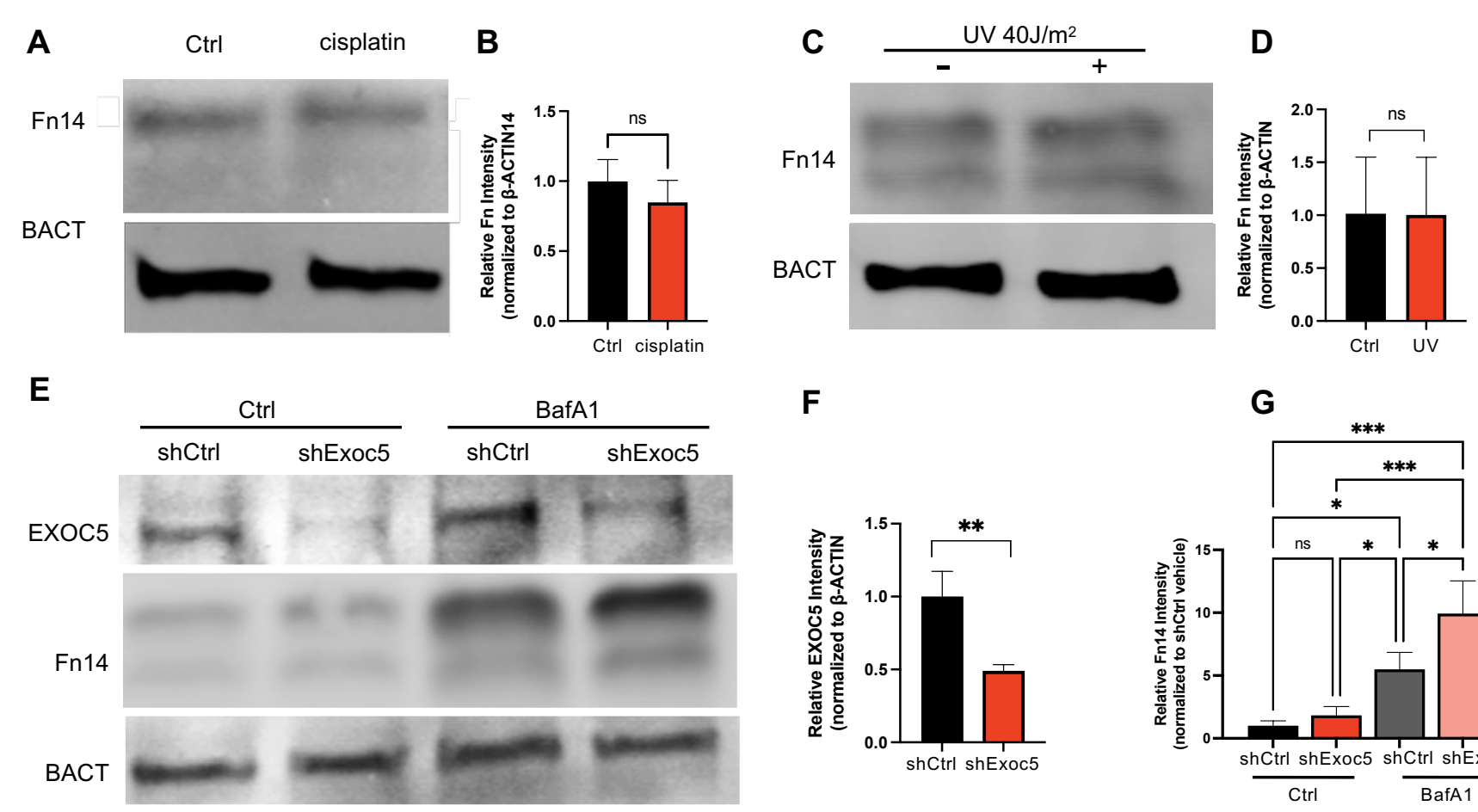

F

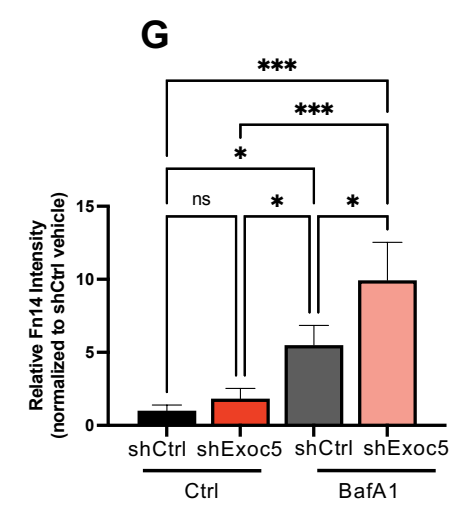

H
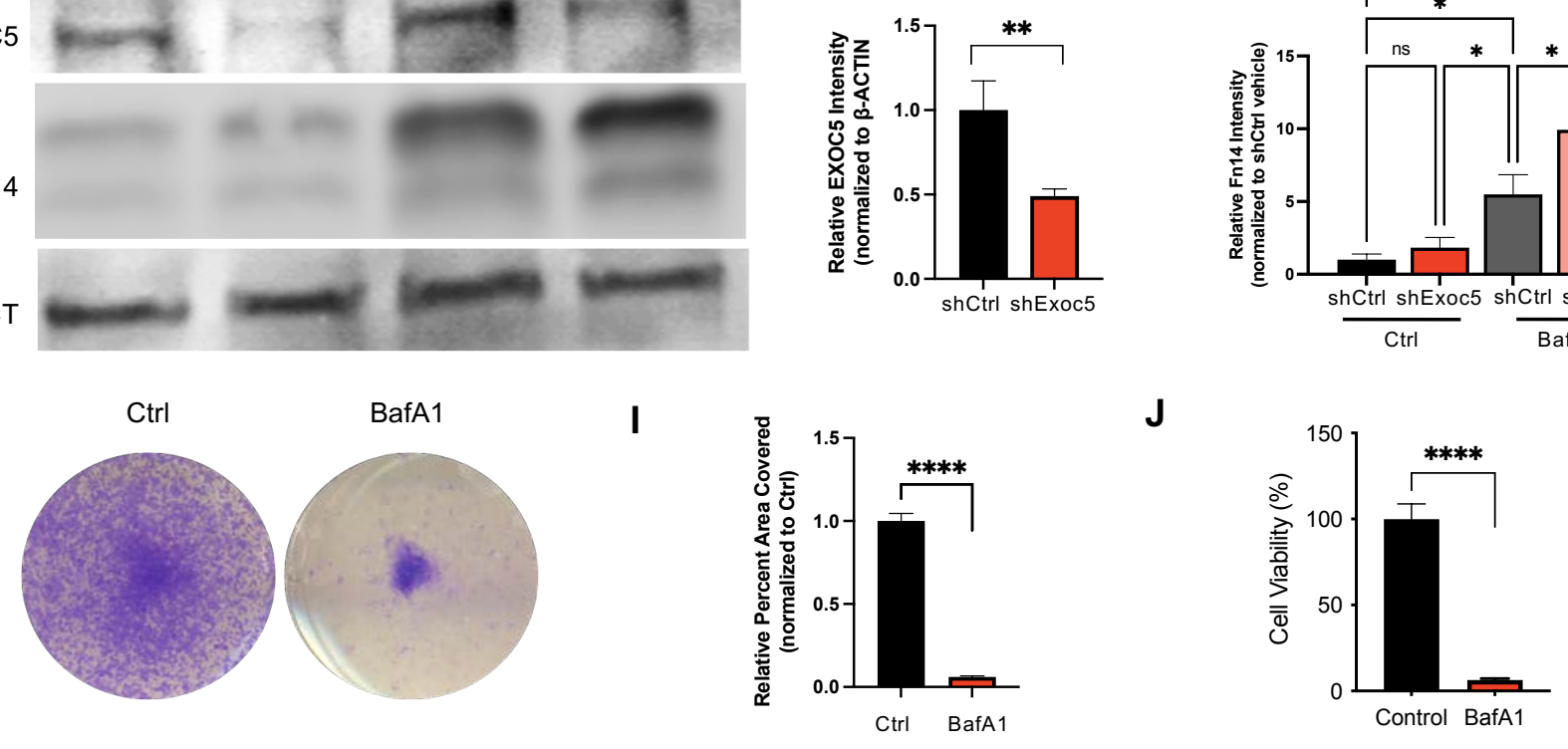

Figure 5 

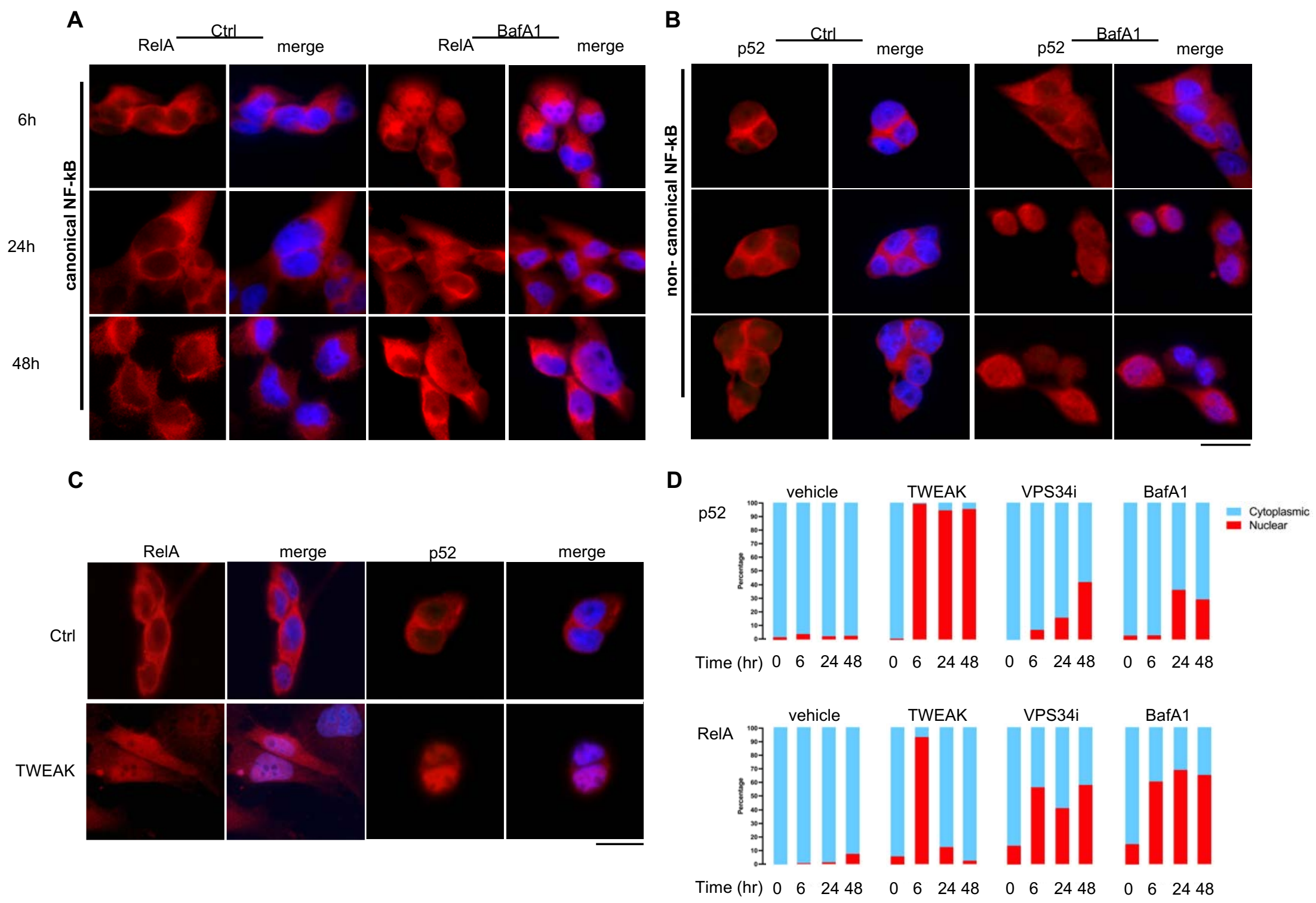

Figure 6 

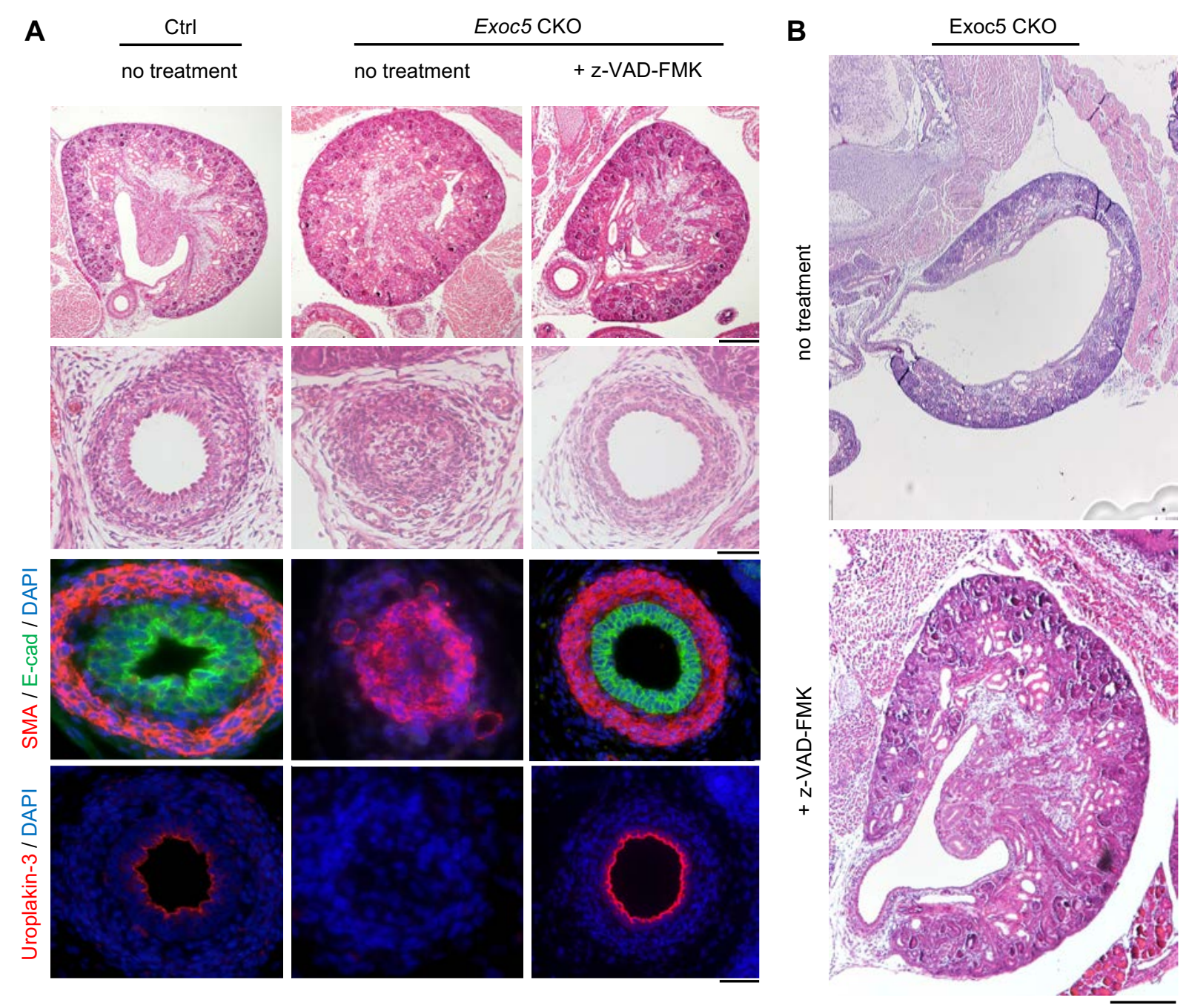

Figure 7 


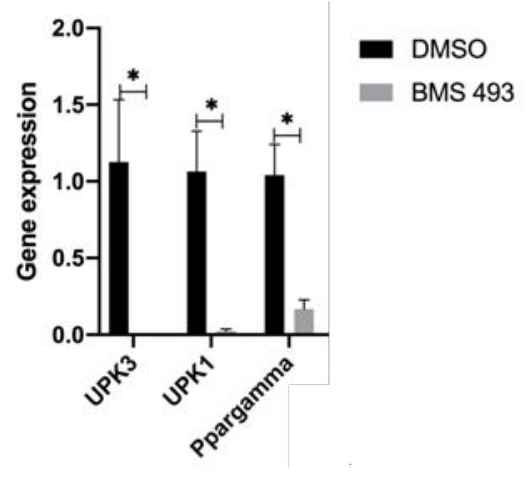


A

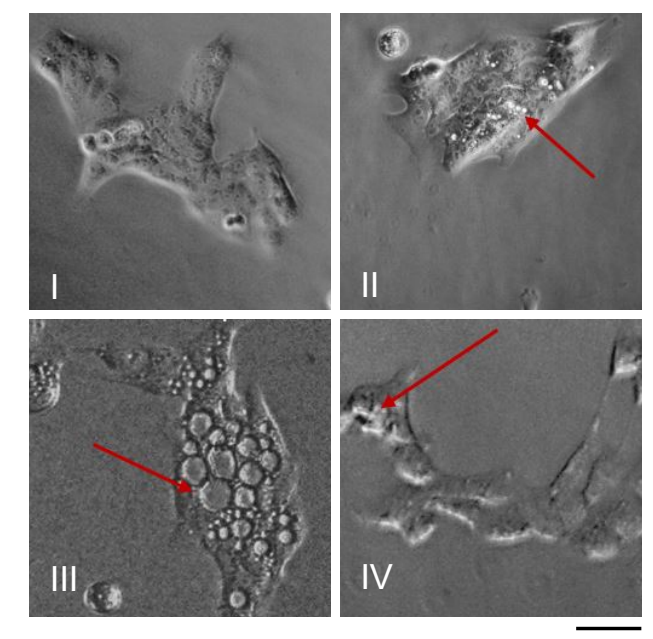

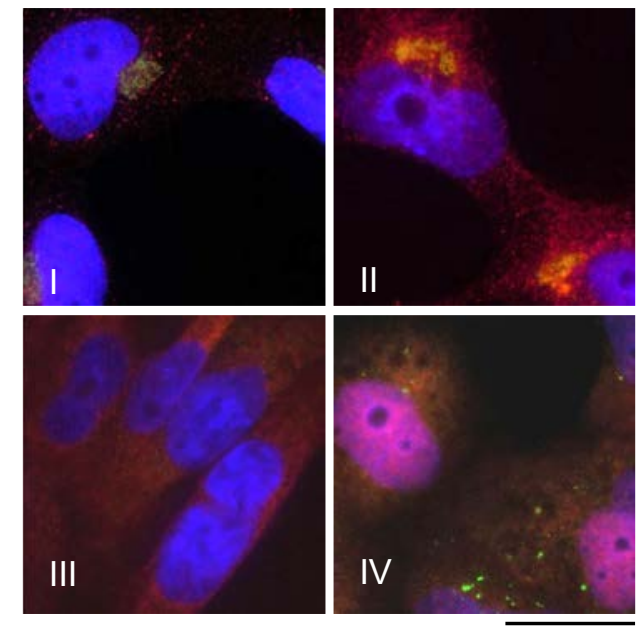

